\title{
WestVirginiaUniversity
}

THE RESEARCH REPOSITORY @ WVU

Graduate Theses, Dissertations, and Problem Reports

2004

\section{Effectiveness of electrical demand reduction strategies}

Chandra Gopalakrishnan

West Virginia University

Follow this and additional works at: https://researchrepository.wvu.edu/etd

\section{Recommended Citation}

Gopalakrishnan, Chandra, "Effectiveness of electrical demand reduction strategies" (2004). Graduate Theses, Dissertations, and Problem Reports. 1540.

https://researchrepository.wvu.edu/etd/1540

This Thesis is protected by copyright and/or related rights. It has been brought to you by the The Research Repository @ WVU with permission from the rights-holder(s). You are free to use this Thesis in any way that is permitted by the copyright and related rights legislation that applies to your use. For other uses you must obtain permission from the rights-holder(s) directly, unless additional rights are indicated by a Creative Commons license in the record and/ or on the work itself. This Thesis has been accepted for inclusion in WVU Graduate Theses, Dissertations, and Problem Reports collection by an authorized administrator of The Research Repository @ WVU. For more information, please contact researchrepository@mail.wvu.edu. 
Effectiveness of Electrical Demand Reduction Strategies

Chandra Gopalakrishnan

Thesis submitted to the

College of Engineering and Mineral Resources

at West Virginia University

in partial fulfillment of the requirements

for the degree of

Master of Science
in

Industrial and Management Systems Engineering

B. Gopalakrishnan, Ph.D., Chair

Ralph Plummer, Ph.D.

Wafik Iskander, Ph.D.

Department of Industrial and Management Systems

Engineering

Morgantown, West Virginia

2004

Keywords: Electric Demand, Energy Conservation, Simulation 


\section{ABSTRACT \\ Effectiveness of Electrical Demand Reduction Strategies \\ Chandra Gopalakrishnan}

A common notion in the industry is that energy is an overhead and not related directly to the product. Measures that reduce electric demand are usually considered in isolation and their effect on the other costs related to the product is ignored. This is especially true for multi-product operations where the demand for various products is not constant and it is hard to track the costs associated with each product. This research takes a software based approach to analyze how a demand reduction measure affects the carrying cost, stockout cost, labor cost, electric usage cost and electric demand cost. This tool can evaluate different demand reduction strategies and their effect on the overall cost. Sensitivity analysis revealed the factors that affect the costs the most. The results of this research suggest that the interactive effects of any demand reduction measure should be considered before implementing them. 


\section{ACKNOWLEDGEMENTS}

I would like to wholeheartedly thank my advisor Dr. B. Gopalakrishnan for his continued support, guidance and encouragement during the course of this research. I wish to express my gratitude to Dr. Ralph Plummer and Dr. Wafik Iskander for their support and constructive criticism. I would also like to thank my supervisor Mr. Darrell Marley and my coworkers at Vermont Energy Investment Corporation for their support in helping me complete my thesis. A special word of thanks goes to my family and friends who made this possible. 


\section{TABLE OF CONTENTS}

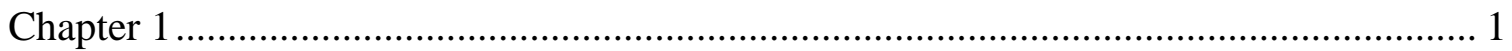

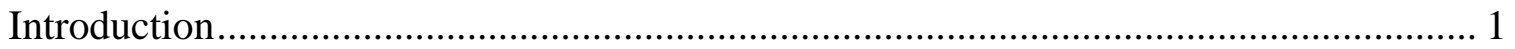

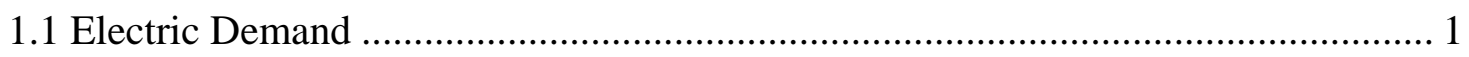

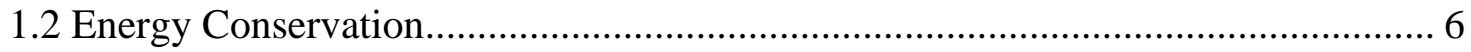

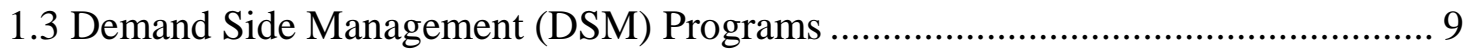

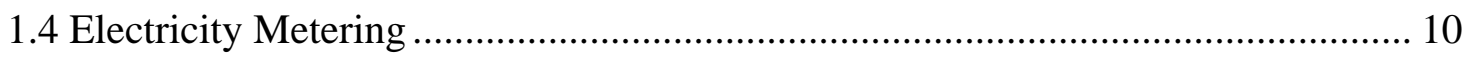

1.5 Strategies currently used to reduce electric costs ............................................. 14

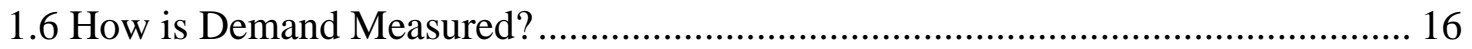

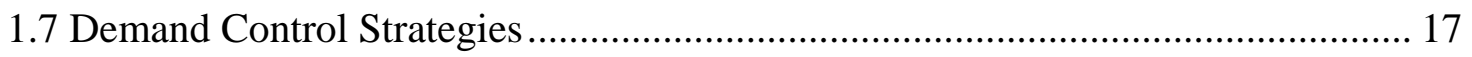

1.7.1 Rescheduling operations (Load Staggering) .............................................. 17

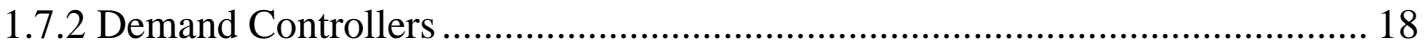

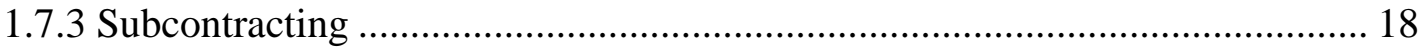

1.7.4 Thermal Energy storage systems ........................................................... 19

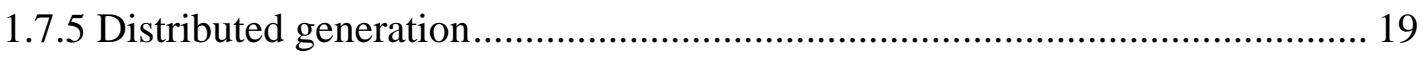

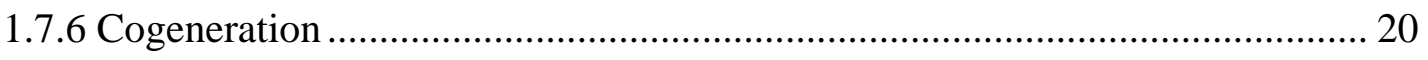

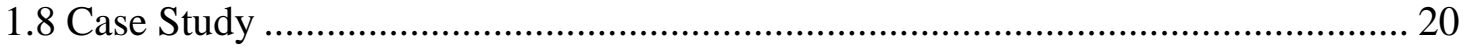

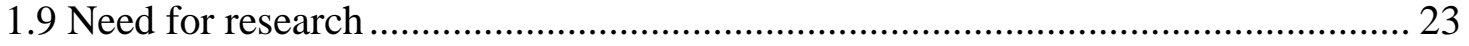

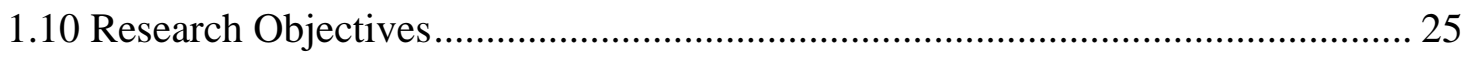

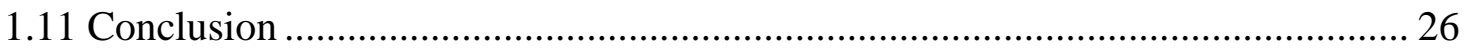

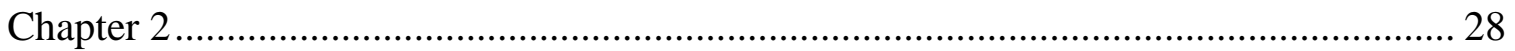

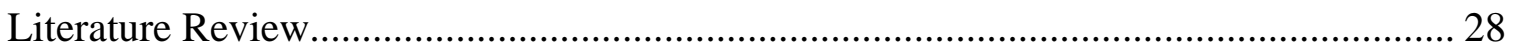

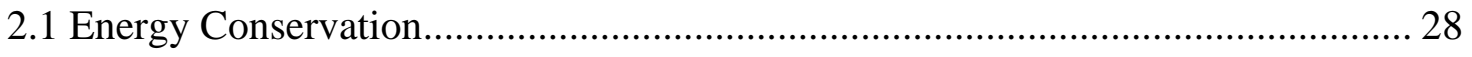

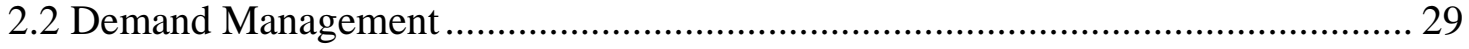

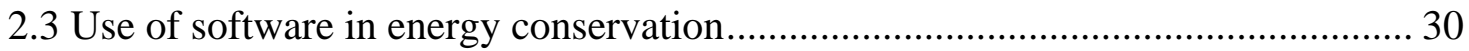

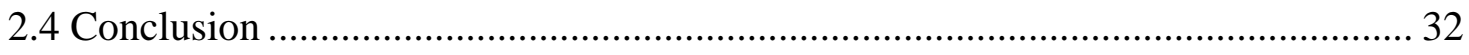

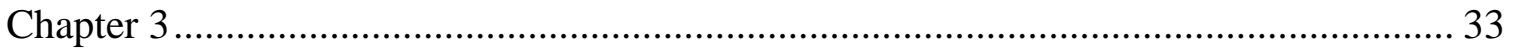

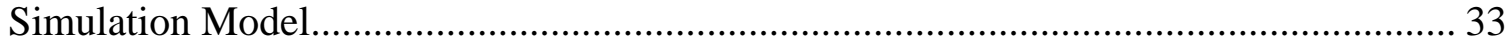

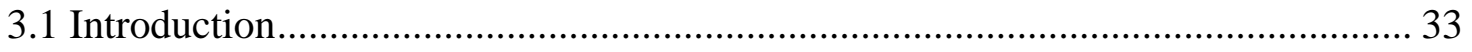




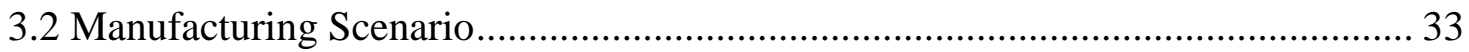

3.3 Operation of the manufacturing facility................................................................ 34

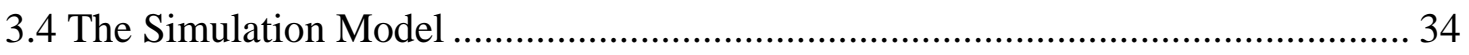

3.5 Production Line 1.............................................................................................. 36

3.6 Production Line 2............................................................................................... 39

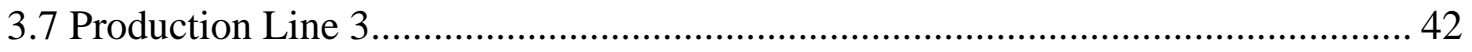

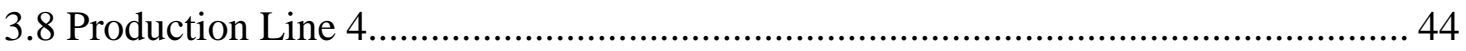

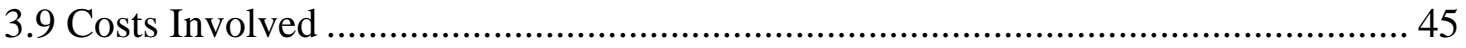

3.10 Inventory Tracking .............................................................................................. 48

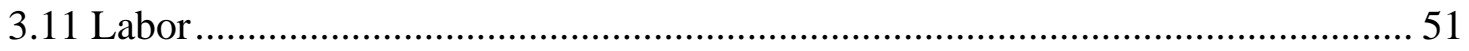

3.12 Energy Usage Monitoring................................................................................. 51

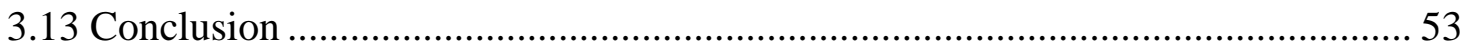

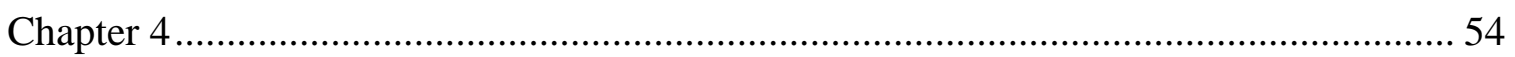

System Implementation .......................................................................................... 54

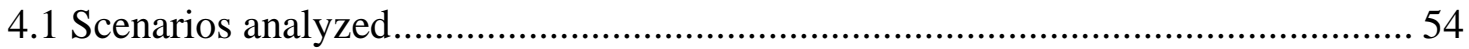

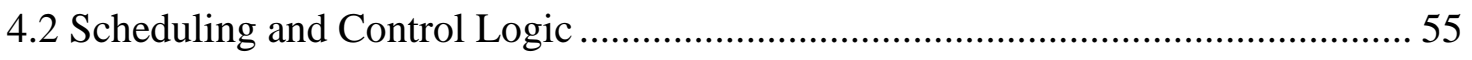

4.3 Scenario 1 Logic (Maximum of 4 production lines at any time)............................ 56

4.4 Scenario 2 logic (Maximum of 3 production lines at any time) ............................... 57

4.5 Scenario 3 logic (Maximum of 2 production lines at any time) ............................. 58

4.6 Input values provided by the user .......................................................................... 58

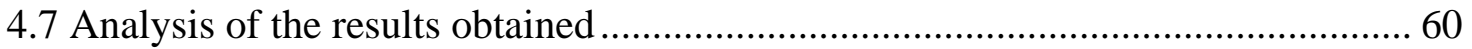

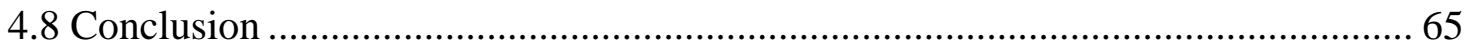

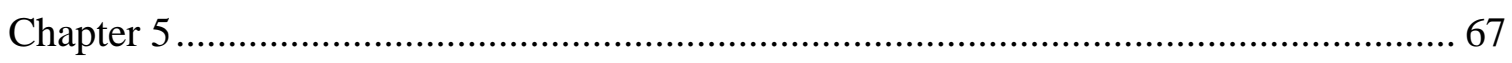

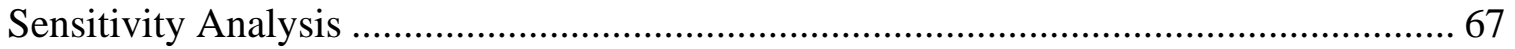

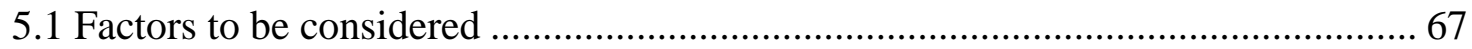

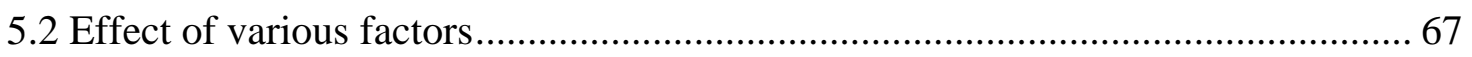

5.2.1 Peak usage cost .............................................................................................. 68

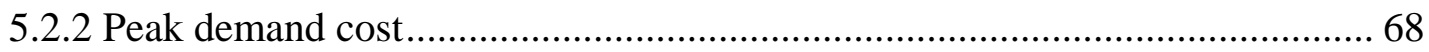

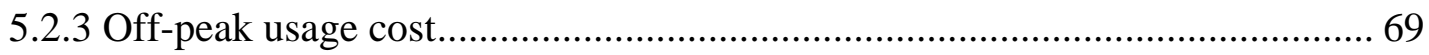

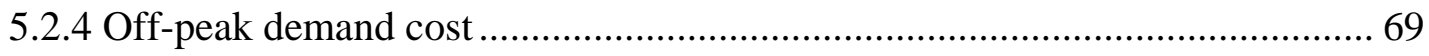




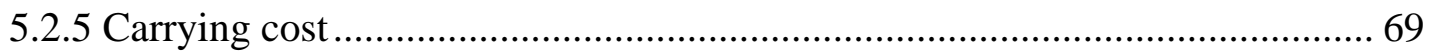

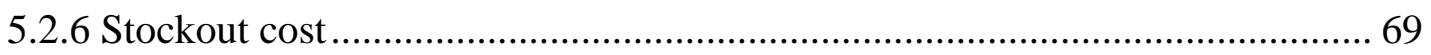

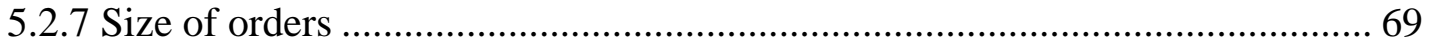

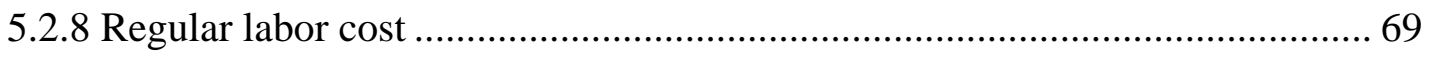

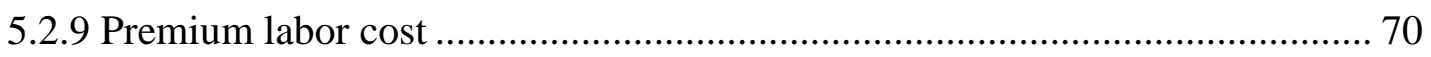

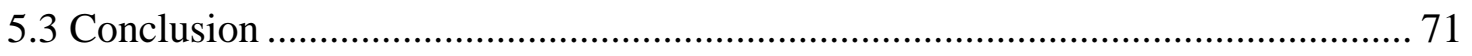

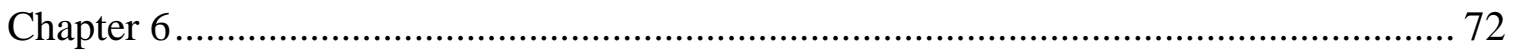

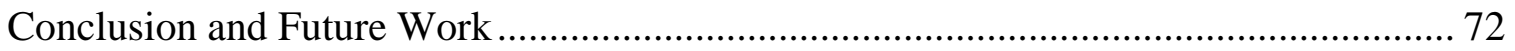

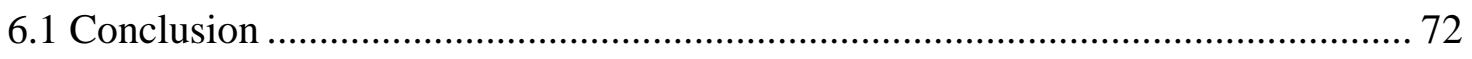

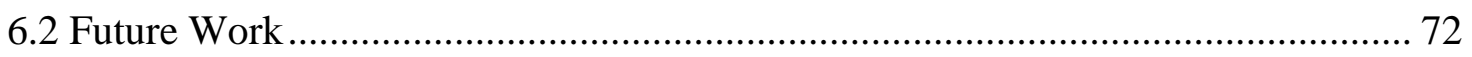

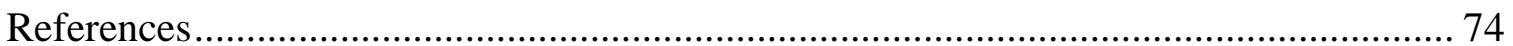




\section{LIST OF TABLES}

Table1.1 Electric Usage Summary of a typical manufacturing facility........................... 5

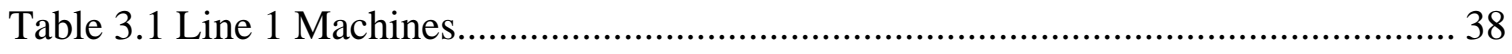

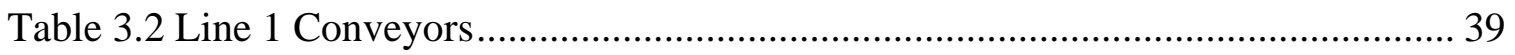

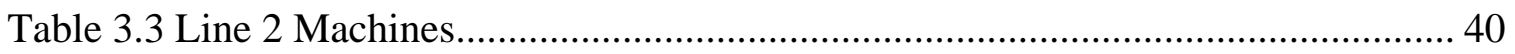

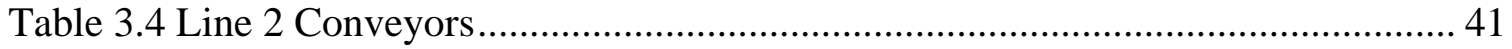

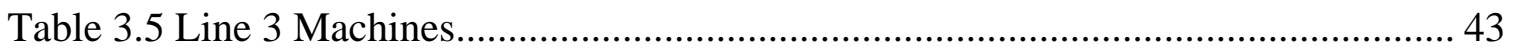

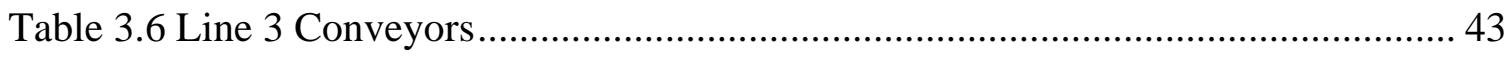

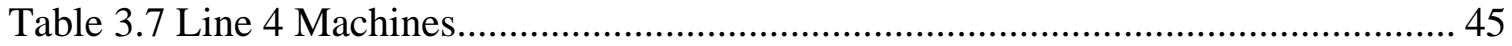

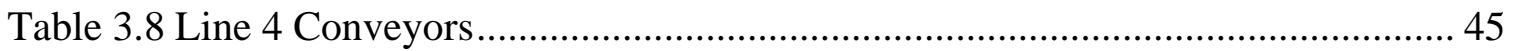

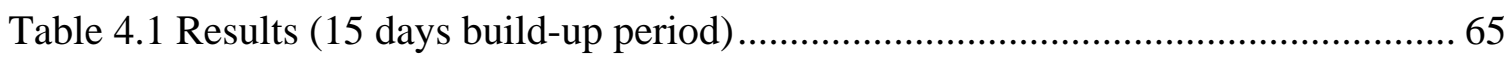




\section{LIST OF FIGURES}

Figure 1.1 Economic Growth and Electricity Consumption......................................... 1

Figure 1.2 Electric bill of a manufacturing facility ..................................................... 4

Figure 1.3 Various components of an electric bill..................................................... 12

Figure 1.4 Calculating electric demand in a 15-minute interval................................... 16

Figure 1.5 Current Assembly and Press Room Operating hours ................................... 21

Figure 1.6 Proposed Assembly and Press Room Operating hours ................................ 21

Figure 1.7 Load profile after moving press operation off-peak.................................... 22

Figure 1.8 Load profile before moving press operation off-peak .................................. 22

Figure 3.1 Different sub-models in the simulation model ............................................ 35

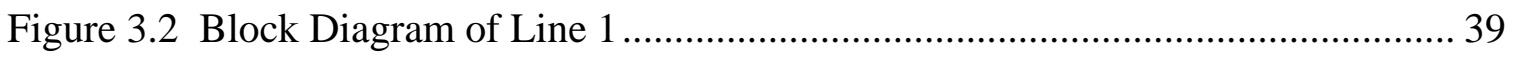

Figure 3.3 Snapshot of the interface showing various costs ........................................ 48

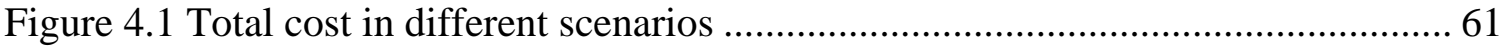

Figure 4.2 Distribution of costs with a maximum of two lines in operation .................... 62

Figure 4.3 Distribution of costs with a maximum of three lines in operation .................. 62

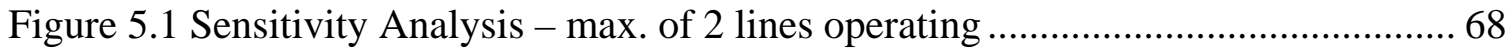

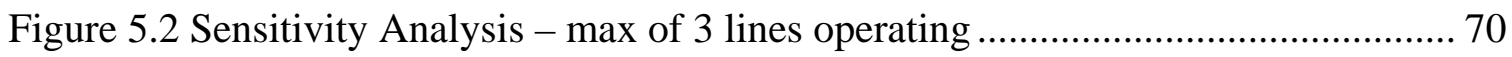

Figure 5.3 Sensitivity Analysis - max. of 4 lines operating ....................................... 71 


\section{Chapter 1}

\section{Introduction}

\subsection{Electric Demand}

Electricity, one of the most extensively used forms of energy, is something that everyone is dependent on in everyday life. Expanding economy, growing population and rising standard of living only emphasize the ever-increasing importance of energy in our lives. The most significant portion of the nation's primary energy consumption occurs in the industry, business and utility sectors - residential use accounts for only 7\% (U.S. DOE, 2003). The availability of energy has an influence on the development of all societies. The fact that electricity consumption in the U.S is closely linked to the economical growth is illustrated by the following graph (Bureau of Economic Analysis, 2003).

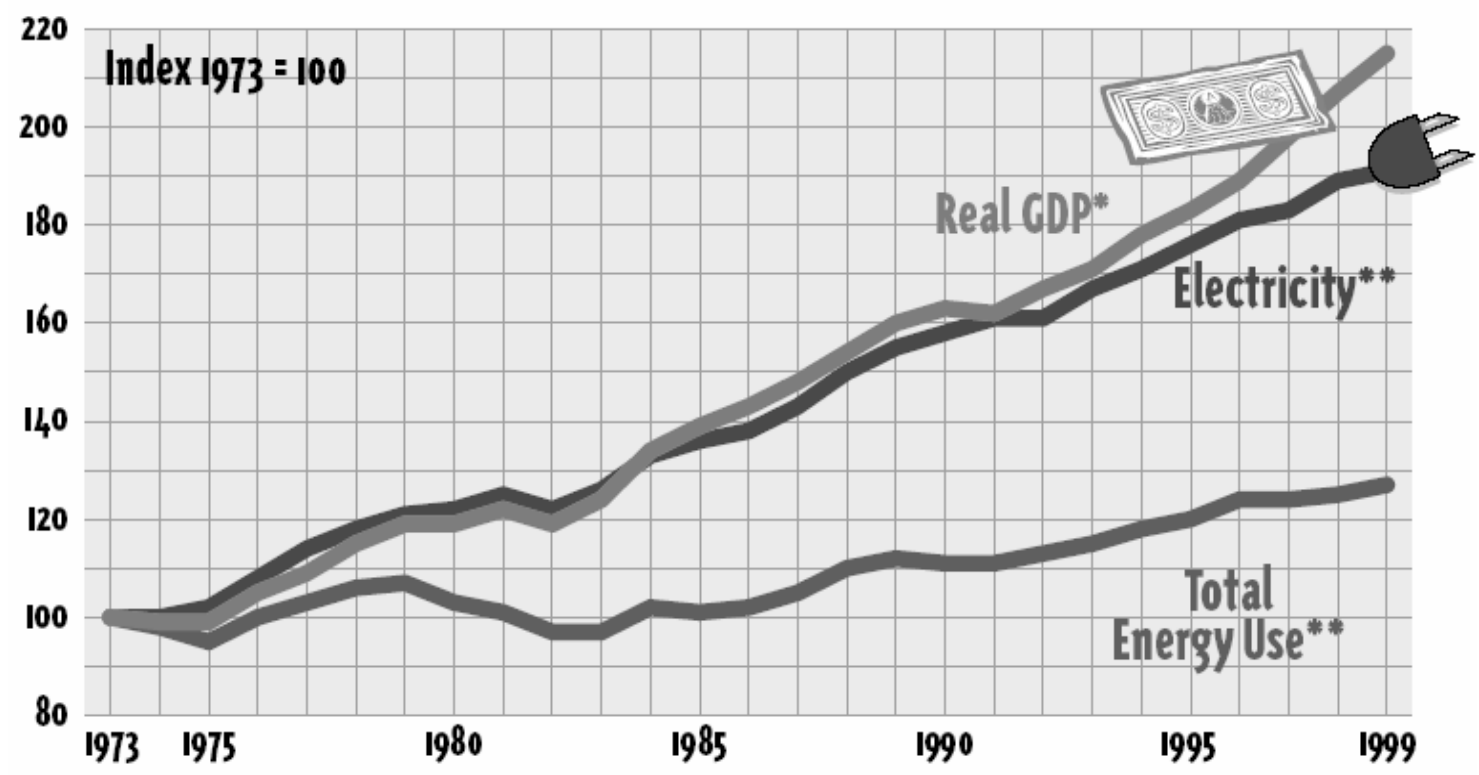

Figure 1.1 Economic Growth and Electricity Consumption 
Electric demand, in simple terms, is the power of the electricity used. For example, five 200-watt lamps need electric power of 1,000 watts or 1 kilowatt. The amount of electrical energy actually used is measured in kilowatt-hours (kWh). Five 200watt lamps when turned on for one hour consume 1 kilowatt-hour. Since electricity is not easy to store, the utilities must have expensive generation and distribution equipment on standby to supply electricity when a customer is using it at a high rate. Even if the customer does not need it for a long time, the utility has to have the capacity to fill the peak demand. Some customers will need it only once in a while, while others need it almost constantly. To spread out the cost of the extra equipment required to fill peak demands, utility companies charge most industrial and commercial customers accordingly.

In the case of residential customers, the electrical consumption and demand are fairly predictable and constant. This enables utility companies to come up with a "blended" usage cost rather than charging residential customers separately for demand.

Electrical demand cost, one of the major components of most electric bills, has received much attention in recent years because of its impact on the operating cost of manufacturing/commercial facilities and utility companies alike. The cost of building new standby generation facilities is passed on to the customer in the form of electric demand cost. This system is both fair and necessary. It is fair because only those customers who use the needed capacity are billed and it is necessary because the service cannot be provided if utilities don't charge for it. Typically, the electric demand cost amounts to $40 \%$ of the total electric cost for a facility. 
The effect and scale of electric demand cost can be highlighted by the electric bill of a facility audited by the Industrial Assessment Center at West Virginia University. This facility follows a rate schedule that charges separately for usage and demand. Also, it has different on-peak and off-peak rates for demand that would allow it to pay less for demand if used advantageously. This facility is a real world example where demand reduction, especially load staggering, will make a difference in the bottom-line.

Analysis of the electric bill, as shown in Figure 1.2, reveals that demand cost was $47.2 \%$ of the total electric cost of the facility for that billing period. The rate schedule is designed to penalize customers with a large on-peak electric demand. Also, a closer look reveals that the rate schedule is so designed that the off-peak demand of 6,418 $\mathrm{kW}$ does not contribute to the demand cost at all. It encourages customers to reduce peak load and shift operations to off-peak hours (10.00 PM to 7.00 AM). A load factor of 61\% means that the average load is less than $2 / 3^{\text {rd }}$ of the maximum load at the facility. The onpeak demand cost per $\mathrm{kW}$ is reduced by a small amount when the $\mathrm{kW}$ is greater than 3000. 


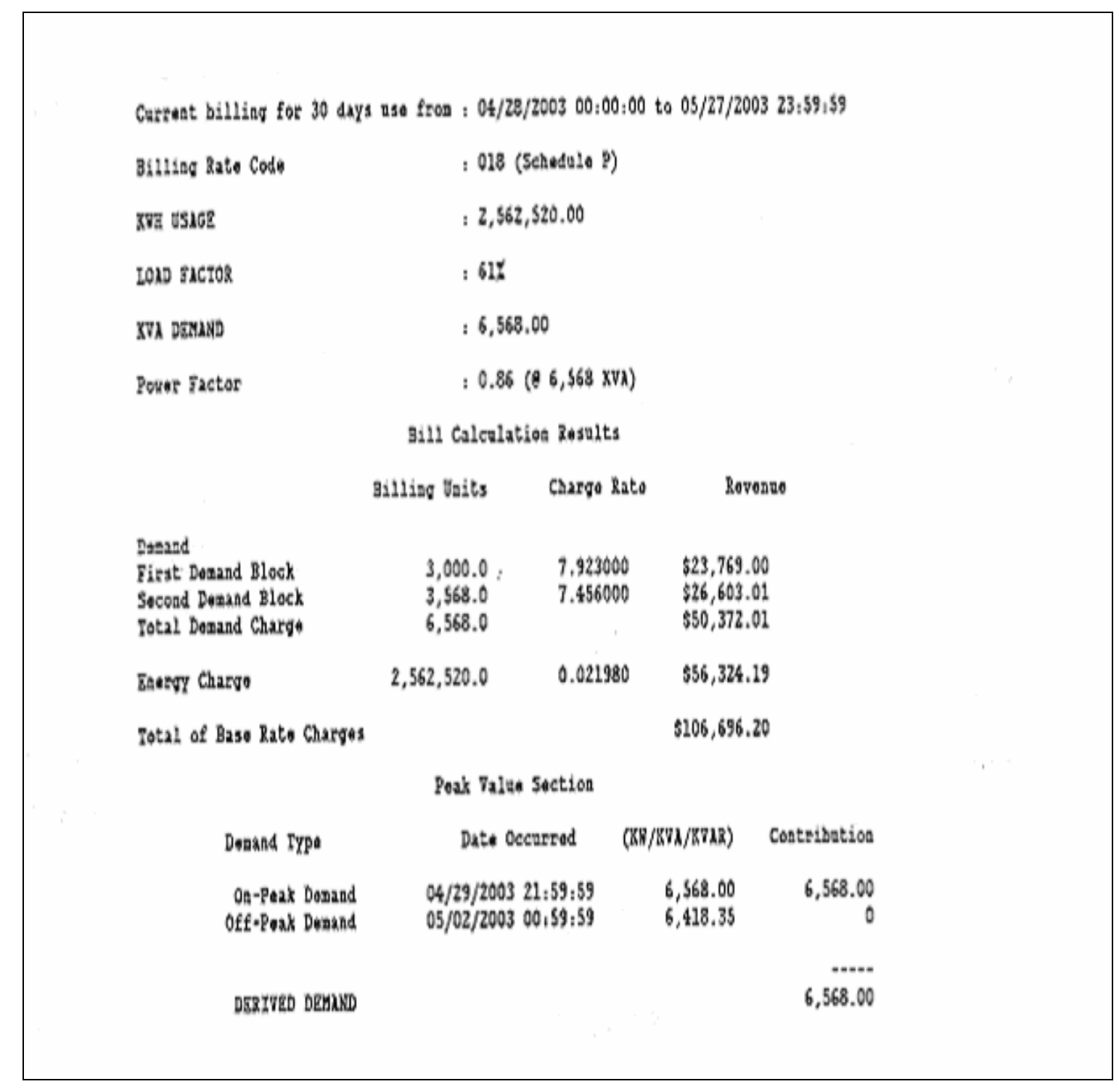

Figure 1.2 Electric bill of a manufacturing facility

The following annual electric cost summary gives a clear idea of the various components of the bill. 
ELECTRIC USAGE SUMMARY

\begin{tabular}{|c|c|c|c|c|c|c|c|}
\hline Year & Month & $\begin{array}{l}\text { Energy } \\
\text { Usage } \\
(\mathrm{kWh}) \\
\end{array}$ & $\begin{array}{l}\text { Billed } \\
\text { Demand } \\
(\mathrm{kW})\end{array}$ & $\begin{array}{l}\text { Usage } \\
\text { Cost } \\
(\$)\end{array}$ & $\begin{array}{c}\text { Demand } \\
\text { Cost } \\
(\$)\end{array}$ & $\begin{array}{c}\text { Total } \\
\text { Cost } \\
(\$)\end{array}$ & MMBtu \\
\hline \multirow[t]{7}{*}{2002} & June & $2,562,520$ & 6,568 & 56,324 & 50,372 & 106,696 & 8,746 \\
\hline & July & $2,390,944$ & 6,846 & 52,553 & 52,442 & 104,995 & 8,160 \\
\hline & Aug & 1,992,222 & 6,152 & 43,709 & 47,270 & 90,979 & 6,799 \\
\hline & Sept & $2,365,582$ & 6,984 & 51,995 & 53,474 & 105,469 & 8,074 \\
\hline & Oct & $2,117,452$ & 6,301 & 46,542 & 48,380 & 94,922 & 7,227 \\
\hline & Nov & $2,071,303$ & 7,063 & 45,527 & 54,060 & 99,587 & 7,069 \\
\hline & Dec & $2,700,619$ & 6,556 & 59,360 & 50,286 & 109,646 & 9,217 \\
\hline \multirow[t]{5}{*}{2003} & Jan & $2,432,840$ & 6,302 & 53,474 & 48,390 & 101,864 & 8,303 \\
\hline & Feb & $3,057,908$ & 7,617 & 67,212 & 58,194 & 125,406 & 10,437 \\
\hline & March & $2,768,155$ & 7,215 & 60,844 & 55,195 & 116,039 & 9,448 \\
\hline & April & $2,781,727$ & 6,340 & 61,142 & 48,668 & 109,810 & 9,494 \\
\hline & May & $2,709,169$ & 7,339 & 59,547 & 56,120 & 115,667 & 9,246 \\
\hline Total & & $29,950,441$ & - & $\$ 658,229$ & $\$ 622,851$ & $\$ 1,281,080$ & 102,220 \\
\hline Avg & & - & 6774 & - & - & - & - \\
\hline
\end{tabular}

(Other Costs may include tax, surcharge, service charge, late fee etc. depending on the rate schedule)

Table1.1 Electric Usage Summary of a typical manufacturing facility

- Average kWh Cost $\quad=\$ 0.04277$

- Average MMBtu Cost $=\$ 12.53$

- Average $\mathrm{kW}$ Cost $\quad=\$ 7.66$

Demand cost contributes to $48.6 \%$ of the total annual electric cost of the facility. Reducing demand charges by rescheduling various operations to off-peak hours may look like a no-brainer in such a case. But the decision may not simply depend on electric demand cost savings. For example, moving operations to another shift will be easier if the operation is $24 \times 7$. Creating an off-shift just for the sake of load management may involve other costs which may make the decision unjustifiable. The effect of the changes on the process, workers and the ability to fulfill customer requests should be considered before 
making a decision. Since demand reduction does not actually reduce the amount of electricity consumed, the benefit is totally monetary. Therefore the impact of a demand reduction measure on the total cost of operating a facility should be taken into consideration.

\subsection{Energy Conservation}

It is clear that there is no way back to the inexpensive energy that was available in the past. With our lifestyle moving towards more dependence on machines, the efficient use of energy is becoming a critical issue worldwide. Uneven geographical distribution of energy resources and exponential growth of population are some of the factors that make the situation worse. The recent power outage that hit 50 million people from New York to Michigan has only highlighted the need for reducing the strain on the grid. Energy saved means less strain on a vulnerable power network and a more reliable supply of electricity. Every kilowatt-hour saved takes some pressure off the grid, boosting the reliability of the entire network.

Industry uses more than one-third of all the energy used in the United States. In the industrial sector, energy conservation is important because lower energy cost helps to improve the profitability of an operation. Energy cost is one of the largest expenditures in running a facility. Energy management-using energy efficiently, ensuring reliable supplies, and reducing costs—is one of the most challenging tasks facing facility managers. Rising energy prices call for increased energy efficiency.

A firm's energy costs have traditionally been viewed as fixed costs, unable to be controlled to an extent that would result in any significant financial benefits for the firm. However, in virtually every business there is now an opportunity to reduce existing 
energy costs, making them variable and therefore manageable. Customers, industries in particular, are realizing that energy is not a fixed cost over which they have little control. Most important, they are learning it is not only how much energy they use, but how and when they use it also count. Allocating and managing energy costs can realize substantial savings. A rather tough economy is making Energy Conservation Measures (ECM) look promising.

At the factory level, the most convincing argument for energy conservation is the guarantee of cost savings accompanied by the obvious benefit of additional funds generated. The energy efficiency initiatives promoted by the government and various organizations such as utilities aim at providing energy that is abundant, reliable and affordable. In recent years there has been a strong movement towards a more efficient and sensible use of the world's non-renewable fuel resources. Parallel to this trend, there has been a major upturn in the search for energy alternatives and a resurgence of interest in more efficient means of satisfying user requirements. Investing money to improve energy efficiency of buildings and equipment provides an immediate and relatively predictable positive cash flow resulting from lower energy bills. In addition to conventional financing options, new options such as performance contracting, where the savings in energy pays for the implementation cost, are making energy conservation projects attractive.

Wise use of energy reduces energy consumption. The benefits of energy conservation measures include:

- Reducing energy bills, thereby saving unnecessary expenditure.

- Maintaining a pleasant working and learning environment. 
- Reducing the emission of greenhouse gases produced from the generation of electricity.

- Reducing the rate at which non-renewable fossil fuels are being used.

The current trend towards energy conservation can be summed up by the following words (Abraham, 2001):

"Developing a stronger array of energy efficient technologies for manufacturing will lower production costs, conserve energy and decrease pollution. By making the most efficient use of our energy supply, we make our nation more secure. Additionally, these technologies will improve U.S. industrial productivity and enhance our economic competitiveness."

Investment in energy efficiency is still not being considered important in the corporate world. Energy efficiency measures receive the least attention even if they have a short payback and a rate of return comparable to other business projects (Joel, 2003). Instances where projects with $25 \%$ yield and a four-year payback period are rejected or postponed can be cited. Willingness to invest in new technology is one of the important factors affecting financial decisions regarding energy projects. Another practical reason is that the operations manager working on the shop floor is primarily concerned about keeping the production line up and running, not about increasing energy efficiency. Cutting costs via technical means is not a high profile concern in most industries [4]. General lack of concern or knowledge about the energy costs of a facility is a critical issue that needs to be addressed. 
The availability of information about new technology is also important. Managers, particularly in small and medium sized facilities, do not have the time or the resources to gather information and analyze the feasibility of new technologies to implement in their facilities. This is definitely the case when there is a failure of equipment and immediate replacement is needed. The only aim in the mind of a plant manager is to replace the equipment with that they know would work. There is little time to analyze new technology replacements. Lack of tools to identify energy use and savings is also an important reason behind the tendency to be satisfied with aging technology.

\subsection{Demand Side Management (DSM) Programs}

There are two primary components of an electricity bill - usage cost and demand cost. Usage cost is determined primarily by how much the equipment in a facility is operated and it is charged in dollars per kWh. Demand cost is determined by how the equipment is operated and is charged in dollars per $\mathrm{kW}$ or dollars per $\mathrm{kVA}$. More machines operated at the same time require the utility to supply a large amount of electricity though it may be for a short period of time. The utility is compelled to acquire the generation capacity to fulfill these peak loads, which requires a large investment. This investment cannot be economically justified under normal circumstances because it is useful only during peak hours. Any customer with high peak load requirements pays a penalty in the form of demand cost. Investing in new generation facilities is not preferred by utilities especially after the deregulation of electric markets.

Demand-side management (DSM) programs consist of the planning, implementing, and monitoring activities designed to encourage consumers to modify 
their level and pattern of electricity usage. These programs focus on reducing the $\mathrm{kW}$ at any point of time rather than the $\mathrm{kWh}$ consumption. Most of the DSM programs are initiated by utilities and government agencies and offer huge incentives on their part to facilities that comply.

In the past, the primary objective of most DSM programs was to provide costeffective energy and capacity resources to help defer the need for new sources of power, including generating facilities, power purchases, and transmission and distribution capacity additions. However, due to changes occurring within the industry, electric utilities are also using DSM to enhance customer service. DSM programs result in better understanding of the customer's requirements by the utility since they work with the customers to see how they can help to reduce kW. DSM refers only to energy and loadshape modifying activities undertaken in response to utility-administered programs. It does not refer to energy and load-shape changes arising from the normal operation of the marketplace or from government-mandated energy-efficiency standards. The fact that facilities that implement DSM programs are entitled to receive incentives only reduces the risk involved in implementing a demand reducing measure.

\subsection{Electricity Metering}

Electricity users can be broadly divided into residential, commercial and industrial. Industrial customers are huge in terms of consumption of electricity. For many industries, the cost of electricity represents a large part of their overall costs, sometimes running into millions of dollars per year. Naturally, these industries are focusing on how to conserve electricity and reduce costs. 
The energy cost is an important factor that affects the economic viability of several energy conservation projects. A good understanding of how electricity is charged is required to develop methods aimed at conserving electricity. Generally, a considerable number of utility rate structures do exist within the same geographical location. Each utility rate structure may include several clauses and charges that sometimes make following the energy billing procedure a complicated task. The complexity of the utility rate structures is becoming even more acute with the deregulation of the electric industry. The following breakup of a typical electricity bill in Figure 1.3 will help understanding how exactly utilities charge electricity.

A typical electric bill consists of most of the following charges depending on the rate schedule.

1. Customer / Service charge

2. Energy charge

3. Demand / Capacity charge

4. Power Factor Adjustment charge

5. Automatic Adjustment charge 


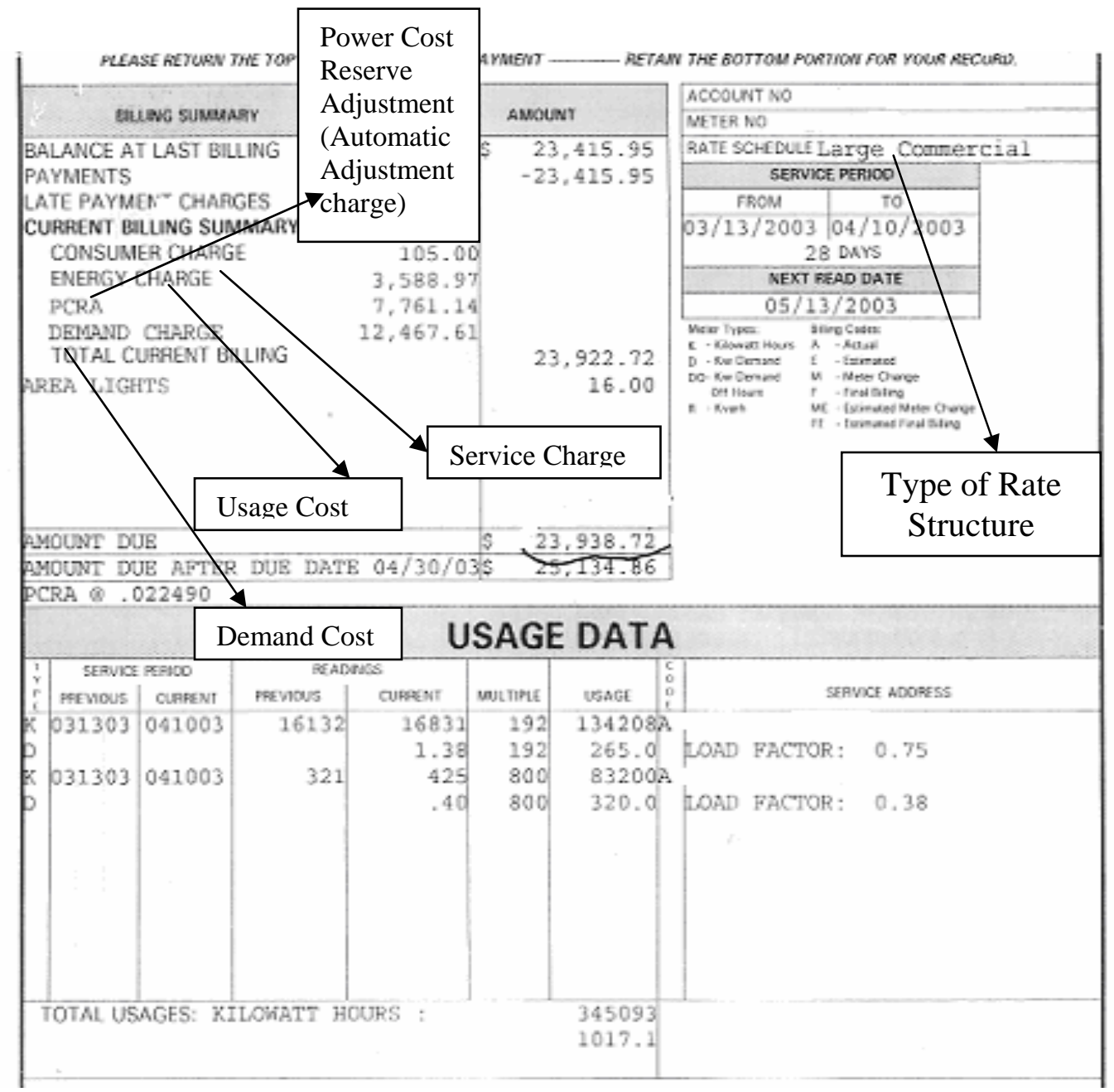

Figure 1.3 Various components of an electric bill

The billing summary in Figure 1.3 shows the charges applied using a Large Commercial Rate Schedule, from March 13, 2003 to April 10, 2004. It also includes a separate charge for the electricity consumed by outdoor lights. Utility companies often provide special rates for exterior lighting. The usage data, below the billing summary, show the meter readings and the multipliers used to calculate the actual $\mathrm{kWh}$ used. The load factors are 
shown for the two meters (main meter and exterior lighting meter). The load factors of 0.75 shows that the average load on the main meter was $75 \%$ of the peak load.

Consumer/Service charge: It is the minimum amount a customer pays even if no energy is used. It is basically the cost for meter reading, meter data processing, postage, bill processing, etc., that is offered to a customer of the utility.

Energy/Usage charge: It is the cost that the consumer pays for the actual amount of electricity used (cost per unit energy). The energy charge is indicative of the utility's cost of producing (or purchasing for resale) a kilowatt-hour (kWh) of energy. It is usually given in dollars/kWh.

Demand/Capacity charge: This charge is not applicable to all consumers. Some utilities have rate schedules in which demand is not billed separately. It is usually included in the energy charge itself for these types of schedules. But it is not equitable to raise the energy charge for all users because customers with constant loads would be paying for equipment used only by other customers with varying loads. For those rate schedules that actually include a Demand Charge, it is related to the maximum demand for electricity that a customer places on the utility's system during the billing period. The demand is measured in thousands of watts or kilowatts $(\mathrm{kW})$ or $\mathrm{kVA}$.

Power factor adjustment charge: It is the cost that a consumer pays if the power factor of the facility goes less than that specified by the utility in the rate schedule. Power factor is the ratio between the KW and the KVA drawn by an electrical load where the KW is the actual load power and the KVA is the apparent load power. It is a measure of how effectively the current is being converted into useful work output and more particularly is a good indicator of the effect of the load current on the efficiency of the supply system. 
Inductive loads such as fluorescent lamp ballasts cause the voltage and current they use to shift out of phase. The utility much supply additional power, measured in kilovolt-amps (kVA), to make up for the phase shift. The rate schedule in Figure 1.3 does not penalize customers who have a low power factor.

Automatic adjustment charge: In figure 1.3, a Power Cost Reserve Adjustment (PCRA) is charged. Adjustment charges usually includes Purchased Power Adjustment and Fuel Adjustment, a mean by which the utility is able to pass certain (variable) cost changes directly to the customer without having to resort to a full-scale rate increase/decrease proceeding. This charge depends on the cost that the utility has to pay to buy the fuel to run its generating plants or the cost of the electricity it buys for resale.

Of all these costs, the costs that can be controlled by the consumer are the Energy cost, the Power factor Adjustment cost and the Demand cost. These costs can be minimized by various methods, most of which require a change in the process or equipment used. Energy Cost minimization usually requires a change in the equipment and/or the process. Installing capacitor banks or switching to better equipments can improve the power factor of a facility. Different techniques used to reduce energy cost are discussed below.

\subsection{Strategies currently used to reduce electric costs}

There are three basic approaches that are followed to reduce electricity usage (Smith, 1981):

- Reduce use - by self denial or regulation or economic pressure

- Increase efficiency - better housekeeping and operational procedures; more efficient equipment; different material 
- Substitute energy form - use other forms of renewable or nonrenewable energy sources.

Techniques used to conserve energy differ in terms of implementation cost, implementation time, complexity, and benefit achieved from it. Some of these techniques are listed below:

- Review historical energy use

- Energy audits

- Housekeeping and maintenance

- Analysis of energy use (engineering analysis, computer simulation, availability studies)

- More efficient equipment

- More efficient processes

- Energy containment (heat recovery and waste reduction)

- Substitute material

- Material economy (scrap recovery, salvage, and recycle)

- Material quality selection (material purity and properties)

- Aggregation of energy uses

- Cascade of energy uses

- Alternative energy sources

- Energy Storage

Though there is a good diversity in the techniques used, the general principles behind them are inter-related. Familiarity with the method used by the utilities to charge 
electric demand will help in appreciating the strategies used to reduce electric demand in industrial facilities.

\subsection{How is Demand Measured?}

Different utilities calculate electric demand based on various methods. The most common method of charging electric demand is explained here (Allegheny Power, 2002). Utilities measure demand with a meter that averages demand every 15 minutes (30 minutes in some cases). The following graph shows the demand calculation for a 15minute period in a small facility.

\section{Electric Demand in a 15 minute period}

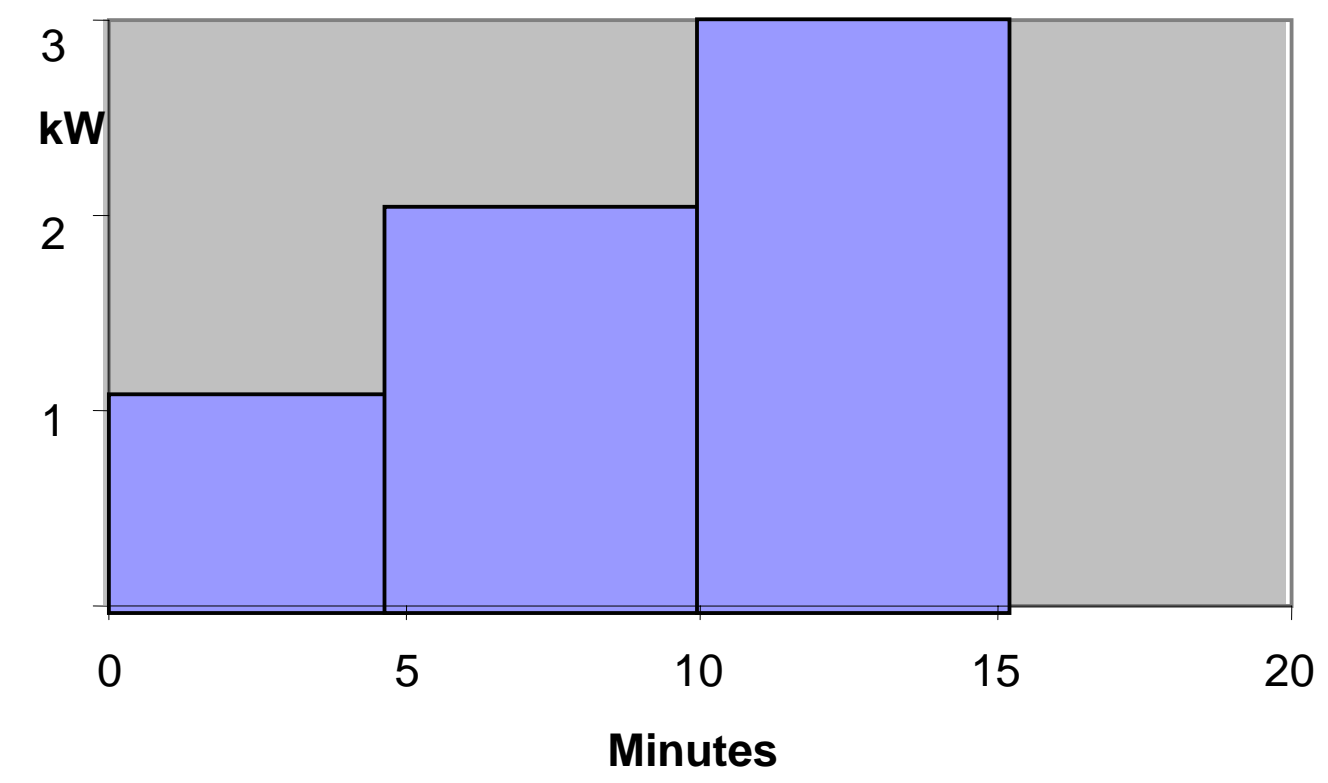

Figure 1.4 Calculating electric demand in a 15-minute interval

The electric demand for this particular interval represented in the graph can be calculated as follows: 


$$
\text { Electric Demand } \begin{aligned}
(\mathrm{kW}) & =[(1 \mathrm{~kW} * 5 \mathrm{~min})+(2 \mathrm{~kW} * 5 \mathrm{~min})+(3 \mathrm{~kW} * 5 \mathrm{~min})] / 15 \mathrm{~min} \\
& =2 \mathrm{~kW}
\end{aligned}
$$

The highest 15-minute average demand in a given month is recorded and charged. Some rate structures have on-peak and off-peak rates also. Utilities have different rate schedules and the user can choose the best rate schedule based on the operating pattern of the facility. Of late, deregulation has given customers more choices than ever. Averaging $\mathrm{kW}$ over a period of time (15 or 30 minutes) prevents customers from being penalized for power surges that occur when equipment are turned on. But on the other hand, the demand cost increases drastically even if one 15-minute period is not controlled. Essentially, demand control requires continuous monitoring in order to be effective.

\subsection{Demand Control Strategies}

The various demand control techniques currently being practiced are analyzed in the following paragraphs. Though the cost and the capacity of the following demand control systems have been discussed, their selection depends on a lot of other factors such as geographical location, utility support, incentives etc. The first three strategies are lowcost options and easier to implement without any external expertise. The other strategies require a high degree of engineering expertise and a huge investment.

\subsubsection{Rescheduling operations (Load Staggering)}

The most important difference between Load Staggering and the other methods used for demand reduction is that the former does not require any investment in terms of equipment. It takes only a careful examination of the energy use profile of the facility to identify the peaks and valleys and how it can be modified to take advantage 
of their current rate structure. Negotiation with the utility may help in securing incentives or even lower rates encouraging load staggering measures. The possibilities of load staggering are unique to every operation and the effects of such a measure should be carefully analyzed.

\subsubsection{Demand Controllers}

These are load-monitoring devices that can monitor the energy use of a facility and cut back non-essential loads to smooth out peaks and valleys. Essentially, these devices switch off "sheddable" loads such as heaters, maintenance equipment etc based on the priorities assigned by the user when the electric demand of the facility goes beyond a preset demand. Some of these devices are integrated with software that allows the user to analyze the energy use pattern using the data collected by the device. Most of these devices do not have any internal decision making capability based on the process. Their capability is limited to shed specific load upon crossing a preset load limit. The cost of a controller ranges from a few hundred dollars for a simple system to thousands of dollars for a complex system that can be networked to an Energy Management System.

\subsubsection{Subcontracting}

Subcontracting certain components or products is a strategy that can help reduce the electric demand due to the reduction in operation of machines. This decision will depend more on the cost that the utility charges the customer for electric demand and the availability of reliable sources of the products. The investment in machines and manpower is reduced by this type of a decision. 


\subsubsection{Thermal Energy storage systems}

Thermal Energy Storage (TES) is generally defined as the temporary storage of energy for later use when heating or cooling is needed. By operating the refrigeration equipment during off-peak hours to recharge the storage system and discharge the storage during on-peak hours, a significant fraction of the on-peak electrical demand and energy consumption is shifted to off-peak periods. Large differential between onand off-peak energy and peak consumption rates generally make cool storage systems economically feasible.

The capacity of these units is usually in ton-hrs of cooling. The sizes range from 3 ton-hrs to 150,000 ton-hrs. The average capacity of the system used in the U.S is about 3000-4000 ton-hrs (Turner, 2001). The cost of the system depends on the cost of the chiller and the cost of the storage tank. The cost of the chiller varies from $\$ 200$ \$1500 per ton. The cost of the storage varies from \$20-\$150 per ton-hr (Washington State University Cooperative Energy Extension Program, 2003).

\subsubsection{Distributed generation}

It refers to a variety of small, local, modular electricity generation units such as internal combustion engines, small gas turbines, fuel cells and photovoltaic cells used to improve the operation of the electricity delivery system. Locally generated electricity from these systems can help meet peak loads without exerting pressure on the grid. The recent trend of utilities purchasing power from facilities having distributed generation capacity is an incentive to invest in this technology. 
The $\mathrm{kW}$ ranges of distributed generation system can range from $20 \mathrm{~kW} / \mathrm{unit}$ for a diesel engine to $30,000 \mathrm{~kW}$ for a gas turbine. The package cost per $\mathrm{kW}$ can be as low as $\$ 125$ for a diesel engine to $\$ 3000$ for a fuel cell (Gas Research Institute, 2000).

\subsubsection{Cogeneration}

The concept of generating both electricity and thermal energy is called cogeneration or Combined Heat and Power (CHP). The electricity produced can be used to reduce the electric demand during on-peak hours and the thermal energy can be used for comfort or process heating. The main advantage of cogeneration is the energy efficiency. If both electricity and thermal energy are fully utilized, a cogeneration plant may have an overall efficiency of $70 \%$ which is significantly higher than the efficiency of 35\% for a typical electric power plant.

The electric capacity of cogeneration units can range from 1-500 kW for a reciprocating gas engine system to $5000-200,000 \mathrm{~kW}$ for a steam turbine cycle system (Turner, 2001).

\subsection{Case Study}

A case study illustrating the cost saving potential of load staggering is discussed below. This energy conservation measure was recommended at a manufacturing facility producing automobile parts.

There were two main operations in this facility: press room and assembly. The

press room operations were at $7 \mathrm{AM}$ to $3.15 \mathrm{PM}$ and $11 \mathrm{PM}$ to $7 \mathrm{AM}$. The assembly operations were at 7 AM to 3.15 PM and 4.30 PM to 1 AM. Presently, the company has an average monthly peak demand of approximately $5,789 \mathrm{~kW}$ and an average monthly offpeak demand of 5,506 $\mathrm{kW}$. The rate structure shows that the peak demand charge is 
$\$ 9.79 / \mathrm{kW}$ where as the off-peak demand charge is only $\$ 1.42 / \mathrm{kW}$. If the company can avoid the combination of press operations and assembly operations during the peak period, it would reduce the peak demand substantially. The off-peak demand rates apply from 11 PM to 7AM.

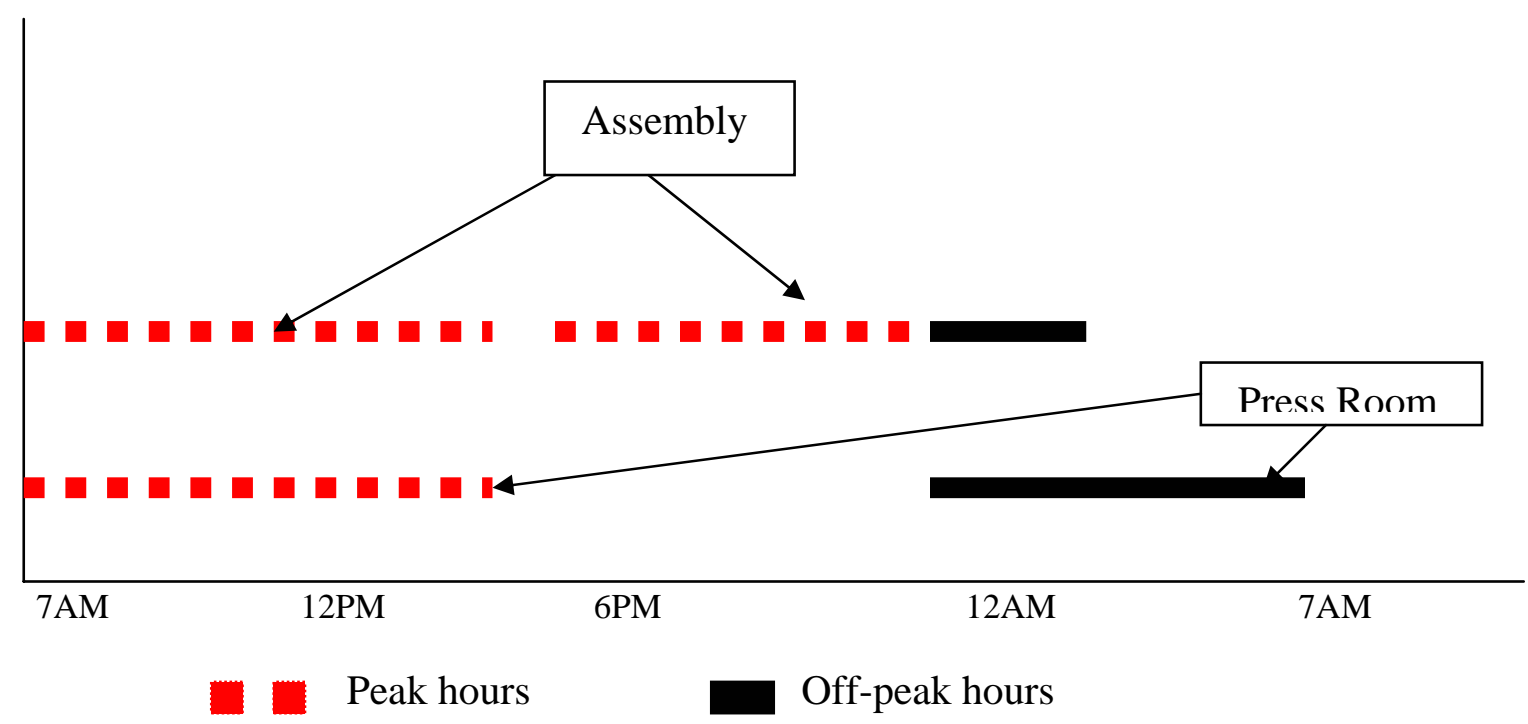

Figure 1.5 Current Assembly and Press Room Operating hours

This energy conservation measure will shift the operations as shown in the following figure.

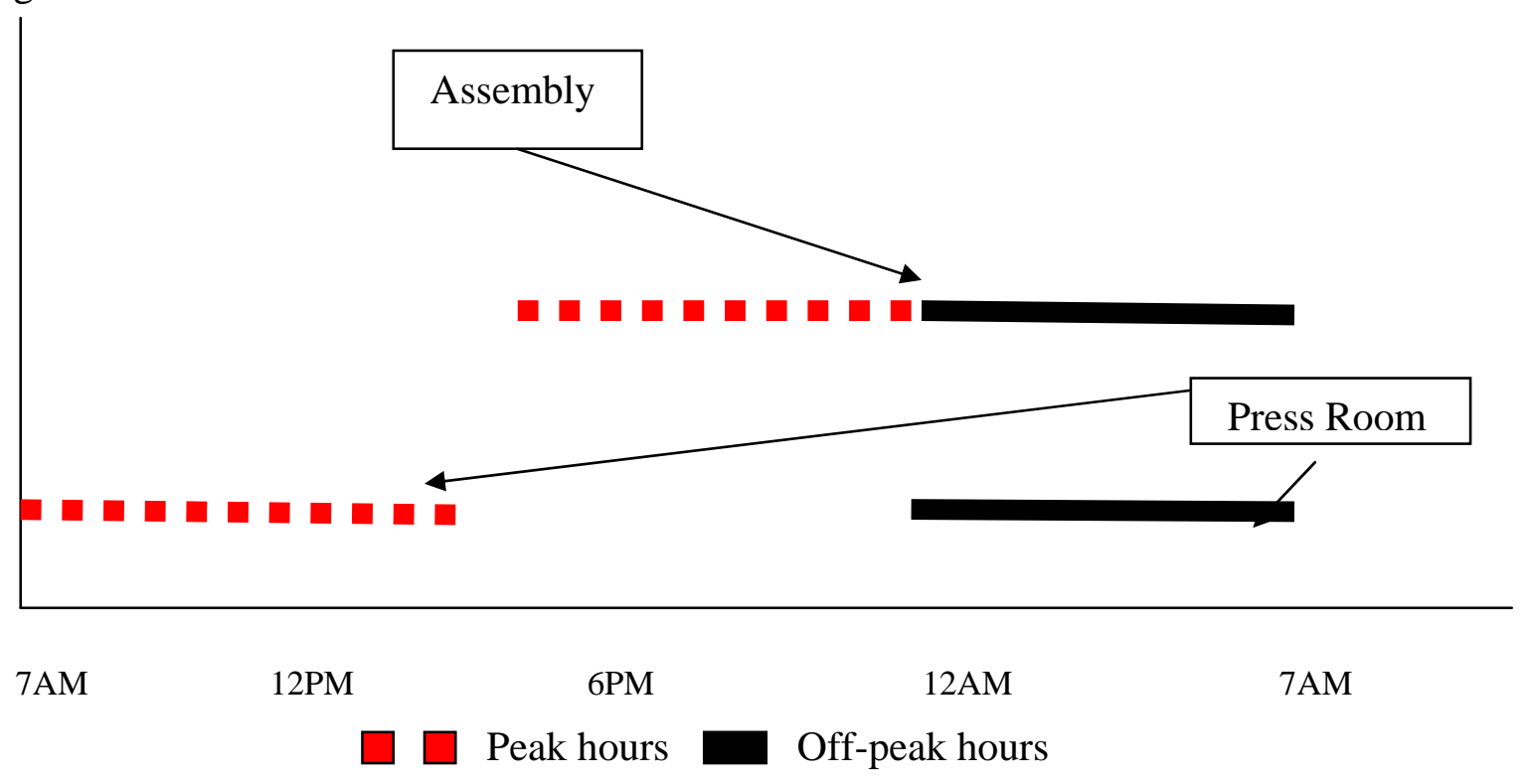

Figure 1.6 Proposed Assembly and Press Room Operating hours 
It can be seen that there is no overlapping of operations during the peak hours. The recommended measure shifts the timing of the press room operation to $7 \mathrm{AM}$ to 3.15 PM and 11 PM to $7 \mathrm{AM}$. The timing of the assembly operation is shifted to $3.15 \mathrm{PM}$ to 7 AM. This load staggering measure involves no cost other than a possible higher labor cost to workers who work off-shift, which would be substantially less than the savings.

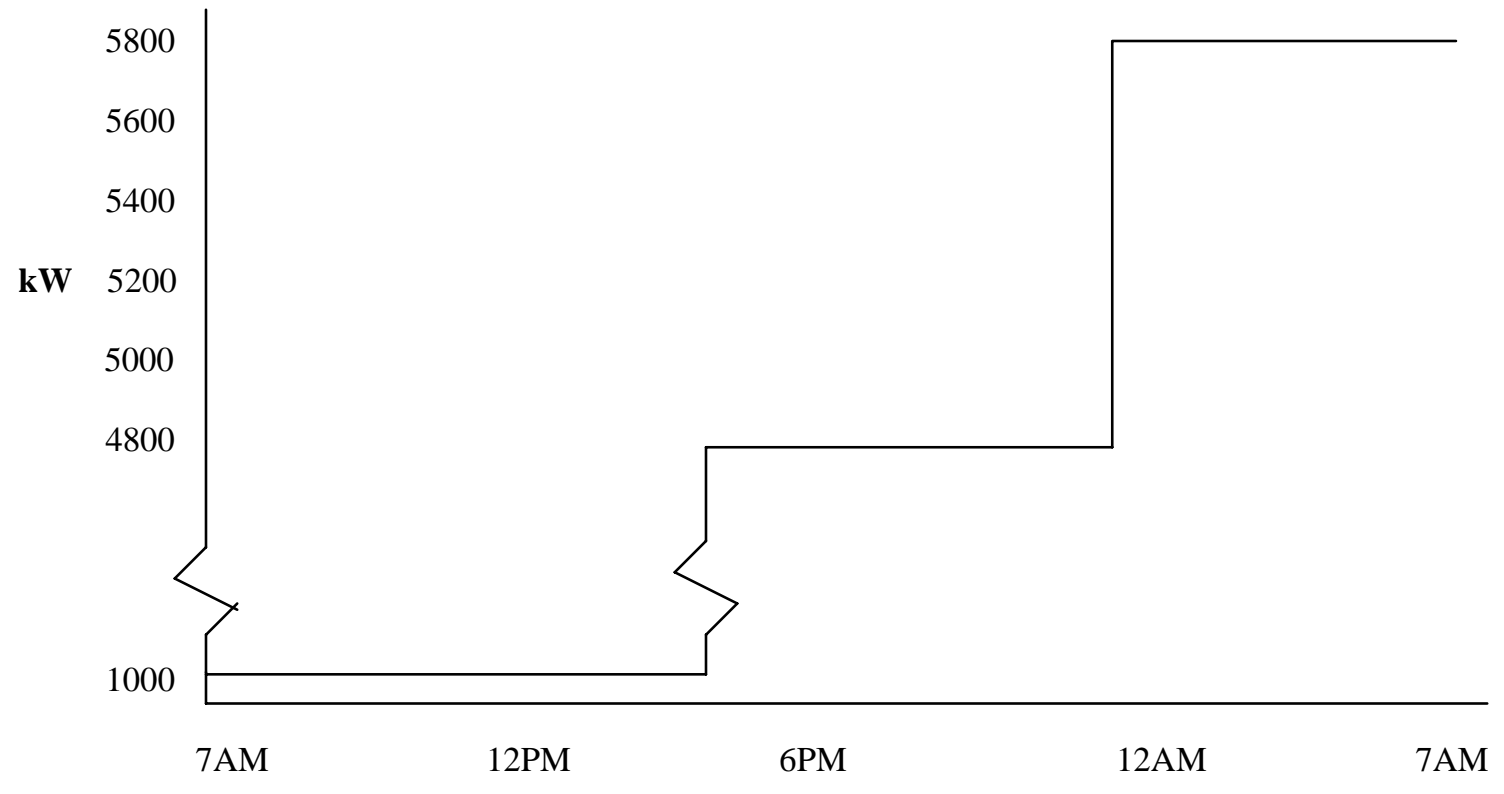

Figure 1.7 Load profile after moving press operation off-peak

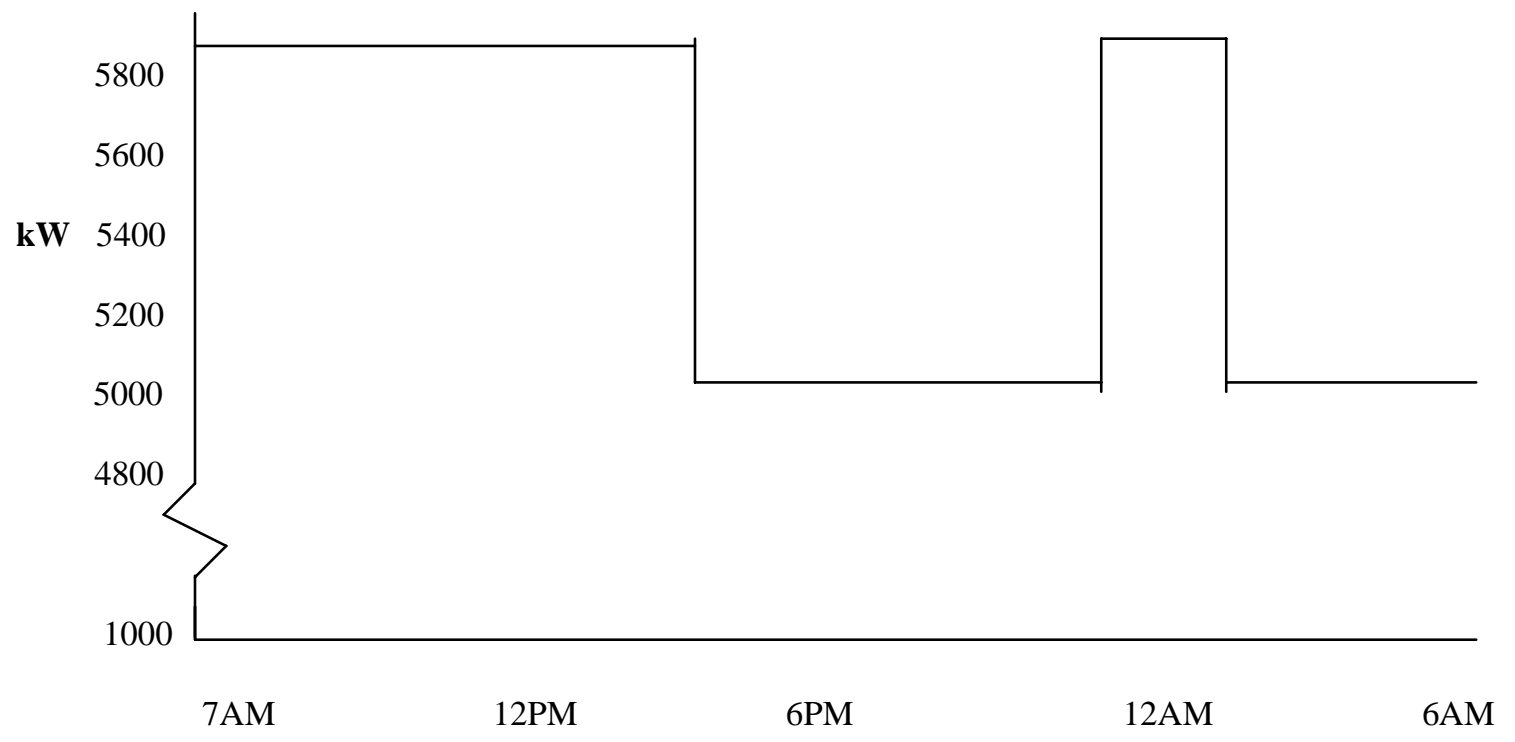

Figure 1.8 Load profile before moving press operation off-peak 
Figures 1.7 shows how the load profile of the operations would change after the changes are implemented. The operations in this facility mostly involved presses and automated processes that need minimal supervision once started by the operators. So they were operated non-stop and the workers took turns when they had to go for a break. The shifts were not exactly 8 hour shifts and were determined by the size of the last product run. The operation was flexible enough to allow shifts that were a little shorter or longer than 8 hours to take advantage of the demand reduction opportunity. Figure 1.8 shows the existing load profile. A conservative estimate of electric demand cost saved annually by this load staggering measure is $\$ 102,000$ (868 $\mathrm{kW} * \$ 9.79 /(\mathrm{kW} . \mathrm{month}) * 12$ months/yr). The fact that this measure needs no investment in terms of equipment or expertise makes it very attractive to implement on the shop floor.

\subsection{Need for research}

Electric demand cost is usually not associated with the products in a manufacturing setting. It is just thought of as a component of the overhead expenses. A reduction in electric demand would mean a reduction in the cost of production meaning a more competitive price for the product in the market. The effect of electricity cost on the cost of a product varies widely depending on how energy-intensive the production process is. As the profit margins get thinner, even a small reduction in costs will help businesses become more successful. Load staggering has been accepted as the most viable method to reduce the demand cost of a facility. Aggregate planning technique to reduce overall operational cost of a facility usually consider costs such as inventory holding cost, profit, labor cost, material cost, overhead and other fixed costs. But the energy costs (usage cost and demand cost) are usually ignored as overhead. The effect of 
demand reduction strategies on the costs mentioned above are usually ignored simply because of their complexity and the unavailability of the right tools. Accurate determination of cost components plays an important role in fixing the price and hence the profit margin of a product (Takukawa, 1997). Also, managers are wary of taking the risk of attempting to find the effects by trial and error.

The primary objective of this research is to identify a methodology to reduce electrical demand without compromising on the product's quality or quantity. But reduction should be done with overall cost in mind. Demand cost is of interest here since it can reduce substantially without even changing the equipment or the process. In many rate structures, demand charges account for $30-50 \%$ of the total billing. The real incentive in reducing demand is that one pays less for the same amount of energy used just by rescheduling operations if possible. Also, the incentives and rebates provided by many utilities make demand saving measures attractive. Merely rescheduling operations can help cut down the energy bills. But the effect of load staggering in conjunction with changes in other parameters can alter the cost savings equation. Since energy cost is sometimes only $10 \%$ of the total operating cost of the facility, the way load staggering affects other major costs should be examined before the implementation of demand saving measures.

The demand controllers available commercially are capable of shedding or cycling loads if the demand goes beyond a preset limit. Usually they are programmed to switch off non-essential loads during periods of peak demand. But their operation is based on the input from the plant personnel, who need a tool like the one proposed in this research, to decide the best demand control strategy. 


\subsection{Research Objectives}

The primary objectives of this research can be listed as follows:

- Modeling a manufacturing operation to analyze the effect of electrical demand reduction measures on the overall cost: The effectiveness of the strategies developed to reduce electric demand cost will depend on the how precisely the real operation is represented in the simulation model. The randomness characteristic of any manufacturing setup should be taken into account for accurate results. Though the model used in this research is not built on real-world data, care has been taken to make it as realistic as possible. The values of most of the parameters used in the simulation model are based on the information obtained from plant personnel, equipment manufacturers, vendors, and are as realistic as possible. The model will be capable of tracking carrying cost, labor cost, stockout cost, electric usage cost and electric demand cost.

- Evaluate different scenarios: Different demand reduction strategies will be evaluated using the manufacturing facility model developed. The costs incurred by using different strategies can be compared to find the most suitable option.

- Sensitivity analysis: One of the several scenarios will be examined in more detail. The values of different parameters can be changed and the effect of this change on the overall cost can be monitored. This would help in finding out the parameters that are of importance to reduce costs.

- Identifying cost saving strategies: The three steps mentioned above will give the user a comprehensive view of the effect of various parameters on the overall cost of the operation. With a dependable tool to analyze alternatives the user can arrive at effective cost saving measures with his knowledge of technical and human factors affecting the operation.

The primary objective of this research is to find the relationships between various parameters and costs and how demand reduction strategies affect these relationships. This means keeping track of different costs accurately by monitoring the operations in the system. At the same time, the tool used to track these costs should allow the user to see what is happening in the system at any point of time and be user-friendly. A graphical 
and visual display of critical parameters and operations would help the user monitor the operations closely. Also, the randomness in the manufacturing operation (operation time, failures, etc.) along with the uncertainty in the demand of various products indicated that simulation software may be one of the options. It was not known initially if the randomness in the operating times or failure times will affect the outcome greatly. The manufacturing operation considered here is not one where every production schedule is perfectly planned in advance. It is one of those operations where the production manager decides which product to produce based on the probable immediate demand for the product. His decision is based on a logic that he has developed from experience.

The level of randomness and uncertainty will vary from one manufacturing set-up to the other. The objective of this research is not to emphasize that the use of simulation software is the only way to evaluate the effect of demand reduction strategies. The real emphasis is on finding out how demand reduction measures affect costs. It may be accomplished using other tools depending on the randomness and complexity of the operation. But it was decided to use simulation because it is customized to represent manufacturing scenarios with relative ease. The simulation model reflects the actual operation with all the uncertainties that one may see in a manufacturing facility. The processing times, number and size of orders, equipment failure are variable. Lot sizes, equipment sizes and the process itself will be realistic. The parameters used will be obtained from the data used in energy audits and discussions with vendors, manufacturers and equipment designers.

The simulation program will not create any production schedule. It will only execute the schedule given by the user. It will accept input from the user regarding the logic and the value of the parameters involved to simulate the production process. The objective is to understand the variation of the costs involved for a schedule provided by the user. The effect of the inventory in stock before the actual orders start arriving will be analyzed to make sure it does not affect the results drastically.

\subsection{Conclusion}

Considering the developing interest shown by manufacturers in energy efficiency as a way to become competent in the marketplace along with the traditional effort to cut 
inventory and stockout costs, this research can be a step to show that these different factors react to each other. It can prove to be a resource that shows where more effort should be concentrated to cut costs. A detailed review of the research done in the areas of energy conservation and electrical demand reduction will be done. The manufacturing operation will be modeled and the effect of various demand reduction strategies will be discussed. 


\section{Chapter 2}

\section{Literature Review}

A review of publications discussing energy conservation programs and demand side management programs (DSM) being implemented widely is discussed here. Also, it analyzes numerous instances where simulation and other software approaches have been applied in energy conservation. These applications exhibit the power and flexibility of these techniques.

\subsection{Energy Conservation}

Corporate practices regarding energy management vary in different companies (Lind, 2000). A survey conducted among 410 companies in the Fortune 500 list is analyzed. The response of the manufacturing sector to energy conservation during the oil crisis and reduced attention towards energy conservation after that period is discussed. Companies now adopt energy management practices for reasons other than just cost benefit. Air pollution, climate change, asset productivity, global competitiveness, shareholder values are some reasons that have still kept energy use in the spotlight for some companies.

The nature and effects of energy conservation programs in the glass manufacturing facilities in West Virginia and Pennsylvania is analyzed by Gopalakrishnan et al (2001). The results of energy assessments conducted at various facilities are summarized. Energy conserving measures discussed in this paper with the cost savings and short payback periods reveals various interesting characteristics of the glass industry. These assessments, performed by the Industrial Assessment Center, WVU - a U.S.DOE project, highlight the benefit of government-sponsored programs to energy conservation.

Energy analysis and diagnostics concentrating on the wood industries are discussed by Mate (2002). Since demand charge is a large part of the energy bills in most of these industries, more emphasis was placed on demand side management. Motors were considered with due importance because of its extensive use in the wood industry. The analysis revealed that most motors were oversized in wood manufacturing facilities. Other energy saving opportunities are also discussed. 
The report prepared by the American Council for an Energy Efficient Economy discusses the soaring electricity costs and rolling blackouts hurting the economy (ACEEE, 2002). The renewed interest in the DSM programs is expected to play to an important role in alleviating the electric system reliability problem. Various DSM strategies supported by various government organizations and utilities and their effects are discussed. Twenty-two case-study programs documented in this report reveal that there are multiple benefits from these programs such as avoiding blackouts, saving energy, reducing customer bills, providing environmental benefits, reducing the market power of suppliers etc.

The need to consider energy conservation projects in light of rising energy costs is emphasized by Heslin (2002). The possibility of new technology or new sources of fuel help reduce the cost of energy is being discussed. The only perceivable short-term solution possible is the conservation of energy. The potential for continued high prices can give a competitive edge to those companies that improve their energy efficiency. Also, higher prices mean a better ROI for energy projects.

The efficiency of industrial processes and the energy systems that propel them are critical to the financial success of a business and can provide it with a definite competitive advantage. The key to making processes and energy systems efficient is to optimize both as a single, integrated system (Smith, 2000). The use of energy to solve operation problems rather than just reduce the use of energy is discussed here. The process is optimized taking energy as a tool to improve product quality, reduce scrap, simplify labor, improve machine utilization, or speed up production. The optimization is done considering the facility in a holistic manner. The idea is to reduce overall costs by improving productivity though it may mean an increase in energy costs sometimes.

\subsection{Demand Management}

Electric supply is essential for any economic growth. The electric demand growth requires the increase of the installed capacity to meet the consumption. Another alternative is the better use of installed capacity (El-Sebaey et al, 2002). Demand Side Management (DSM) has been increasingly adopted by utilities as a substitute for huge investments and as a method for optimization of resources use. DSM is the planning, 
implementation and evaluation of utility activities that are designed to encourage consumers to modify their electricity consumption patterns with respect to both the level demand and energy.

Most of the demand controllers available in the market are designed such it can shed loads based on the priority set by the plant personnel when the demand goes beyond a preset limit. The loads shed are usually non-essential, "sheddable" loads such as electric heaters, air conditioners, exhaust fans, pumps, snow melters, compressors and water heaters etc (Thumann, 1998). They monitor only the loads that they are connected to and reduce or cycle loads so that the demand is kept below a preset limit. Also, minimum on and maximum off times can be entered for each load to protect equipment and maintain comfort. Some of these demand controllers can be remotely operated and can report information in a useful manner. The newest range of these controllers uses neural networks to understand the energy use pattern of the facility it controls.

Demand Side Management is analyzed as a technical and an organizational problem by Nilsson (1994). Technological and economic factors that influence decisions regarding demand side management are being discussed. Various methods of evaluating direct and indirect economic implications of a demand side management program are being discussed by Levine et al (1994). Savings associated with efficiency improvement activities done independent of the utility, but as a result of the participant's positive educational experience in the DSM program and their effect on program benefit evaluation is also discussed.

\subsection{Use of software in energy conservation}

Various Artificial Intelligence and simulation techniques have been used in various energy applications to optimize the use of energy. Some of these applications are discussed below. A demand side management system using the expert system technique is discussed by El-Sabaey et al (2002). It is composed of a main module which, based upon set of rules, selects one of many possible solutions to perform DSM. The system has been tested using data from different industrial, residential and commercial applications. It uses practical experience gained during implementation of energy conservation systems. It allows the addition of extra rules upon user needs. The system 
performs DSM according to one of the following strategies [1-3]: 1- Peak clipping. 2Energy conservation: a) Building automation b) Efficiency equipment. 3- Valley filling. 4- Load shifting. 5- Load building. 6- Flexible load shape.

An attempt to evaluate the waste heat recovery opportunity, and to estimate the space heating and cooling loads, so that a computer based tool in the form of a rule-based expert system is available for energy auditors as well as industrialists to use for decision making is made by Chillarige (1999). The research details on the various aspects involved in the estimation of heating and cooling loads, economizer usage on the airconditioner and heat recovery.

An offline simulation based costing solution to analyze economical process alternatives using Access database is discussed by Feldmann et al (2003). The tool developed in this research makes it possible to make a good decision using simulation and economic data.

Optimization of process parameters using simulation is done by Achariyaviriya (2002). The author analyzes the process of longan drying in a simulation model. The operating parameters drying air temperature, airflow rate and fraction of recycled air are optimized to minimize the energy consumption of the process. The scope of such research is limited to a particular process. Also, the strategy to minimize energy cost is fixed - change the values of a few parameters until an optimal value of the objective function is obtained.

The application of simulation to Activity Based Costing has been analyzed by Takakuwa (1997). A framework to design simulation models is described for a Flexible Manufacturing System (FMS) before actually starting manufacturing in the shop floor. In this study, a simulation model for a FMS is constructed and procedure for cost accounting is developed for different products using ABC. But this study classifies electric costs as a fixed overhead, which is not really true. Also, scheduling of operations is made on the basis of scheduling rules such as LWKR (Least Work Remaining), MWKR (Most Work Remaining), SPT (Shortest Processing Time) etc. and not based on demand minimization or energy conservation. 


\subsection{Conclusion}

The review of the research found in literature underlines the importance of energy conservation throughout the world. Improved technology has provided many tools that have improved the quality of research. Though a lot of topics have been considered in isolation, focus towards overall system improvement is increasing. Energy conservation is the need of the hour. Increased competitiveness in the market has forced manufacturing facilities to reconsider their approach in using energy. Identifying where the dollars are going exactly has become a priority.

Literature shows that electric usage and demand have not been considered as resources that can be directly tied to the product. They have been considered as overhead, sometimes fixed, giving a distorted idea of the exact cost of products. It can be asserted firmly that an approach that allots electricity directly to the product will give a clear idea of the product costs and help in decision-making. Those attempts that have been focused at cutting energy cost have not considered other costs which make up a big chunk of the total cost. Since energy cost makes only a relatively small percentage of the total operating cost of a facility, more effort should be concentrated on finding out how it affects the other costs. 


\section{Chapter 3 \\ Simulation Model}

\subsection{Introduction}

The sections in this chapter explain the manufacturing setup and how the operations are represented in the simulation model. The structure of the simulation program and the logic used will be discussed briefly. This chapter will also show how the user can change the input given to the model to customize it. Tracking of various costs will also be analyzed.

\subsection{Manufacturing Scenario}

The manufacturing scenario considered for analysis in this research is a facility where tinplate cans and lids of three different sizes (large, medium and small) are produced and then filled with paint. The cans are 'drawn and redrawn' two-piece type tinplate cans with slip lids. There are four different production lines in the facility denoted by Line 1, Line 2, Line 3 and Line 4.

The equipment, kilowatts ratings and the speed values used in the model are typical values obtained from observation made during energy audits conducted at facilities with similar operations. Information obtained from discussions with Original Equipment Manufacturers (OEM), can manufacturers and vendors also support the rationale behind the values of the parameters used. Literature from various can manufacturers and professional organizations related to this field has been consulted to arrive at realistic estimates. It should be noted that although similar can-making processes may have variations from facility to facility, care has been taken to represent a generic version with emphasis of operations consuming a comparatively large amount of 
electric power. Some of the operations that typically use natural gas (e.g. ovens to dry the paint) have not been shown in the model, but the process times have been modified accordingly.

Although typical values have been used to analyze the effect of various strategies, the values of the parameters can be easily changed by the user to customize the model.

\subsection{Operation of the manufacturing facility}

The facility can be compared to a small operation with four different production lines. Line 2 produces large and medium cans. Line 3 produces small cans. Line 4 produces lids in all three sizes. Once there is enough cans and lids of the same size, line 1 is operated to fill the cans with paint and seal them with the lids. The filled cans are then shipped to the customers. Orders arrive every week and vary in number and size.

The production schedule is not planned in advance. It depends on the inventory in stock and the orders received or expected. The plant manager uses his experience to use the production lines to a maximum possible extent to prevent a stockout situation. The cans and lids are moved in pallets. Each pallet has a hundred cans in it.

\subsection{The Simulation Model}

The simulation model is divided into seven sub-models for the sake of clarity and simplicity of logic. Four of these sub-models are used for the four different production lines explained above (Steel Cans Production, Lid Production and Can Filling). Tracking energy usage and shipping are done using two different sub-models (Energy Meter and Inventory). The logic to decide the lines to be operated every shift is contained in the sub-model called "Control Logic". Tracking the electric usage and electric demand (both off-peak and peak hours) is done using the "Energy Meter" sub-model. Tracking both 
Work In Process (WIP) and finished goods inventory is done by the "Inventory" submodel.

Animation is used to visualize the operations in the facility. The user will be able to see the complete operation in a single frame. The inventory status is also displayed alongside the animation. The number of various products in the WIP inventory and the finished goods inventory can be seen real-time. The energy meter is also visible in the same screen showing the energy consumption during on-peak and off-peak hours and the associated usage and demand costs. These displays enable the user to know the operating conditions of the facility at any point of time during the production run. All the units related to the number of pieces produced are expressed in number of pallets for convenience.

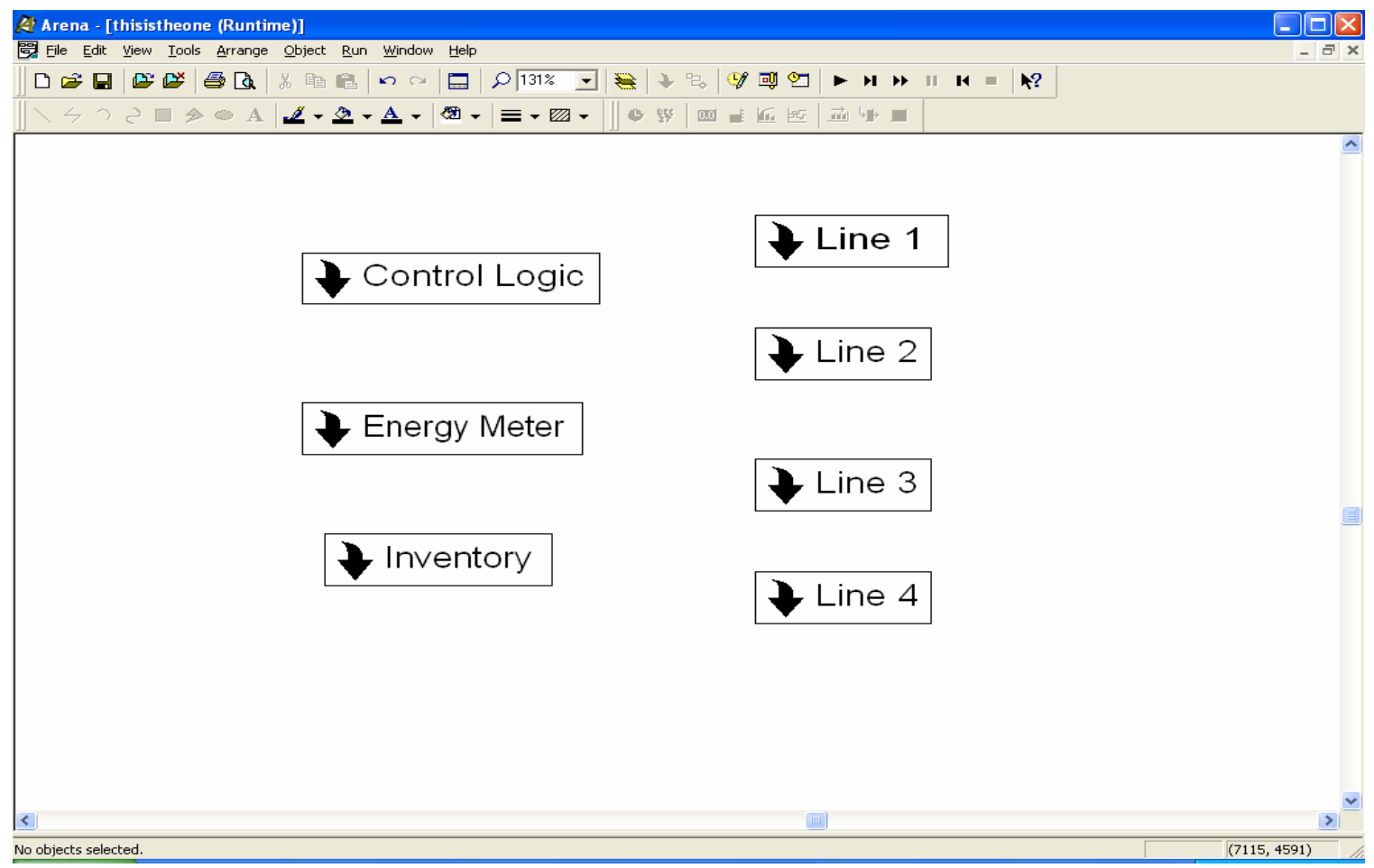

Figure 3.1 Different sub-models in the simulation model 


\subsection{Production Line 1}

This production line is contained in the sub-model named Line 1. It receives pallets of cans and lids produced in Line 2, Line 3 and Line 4 . The pallets are depalletized into individual cans and lids. The cans are then filled with one of three different paints (Red, Green and Blue) in a filling machine. The filled cans are then closed with the lids obtained after depalletizing the lid pallets obtained from Line 4 . This operation is done in a stamping machine. The closed cans are sent to a pre-wash machine before being sent for packing. The groups of cans are then palletized using a palletizer and sent for shipping. Every pallet has 100 pieces or 10 groups of cans. The machines in this line are connected by conveyors. All conveyors are accumulating type conveyors assumed to be operating at a load factor of $85 \%$ (i.e. consuming $85 \%$ of their rated $\mathrm{kW}$ ) when there are products on them and at 50\% load when there are no products on them.

It should be noted that the load factors of the machines are different when processing different sizes of cans. To take the difference in power consumption into account, the load factors of the machines while processing large, medium and small cans are fixed at $85 \%, 65 \%$ and $50 \%$ respectively. These values can be easily changed by the user to represent a particular facility. A load factor of $10 \%$ is used when the machines are idle. The setup time when changing the size or color of the product is given in the form of a triangular distribution.

In this sub-model, an entity is created at the beginning of the shift. This entity checks if the production line is to be operated for the current shift, using the results from the control logic sub-model. Depending on whether the machines are empty and what product is to be produced, the entity signals the release of can and lid pallets from the WIP inventory to the depalletizer machines. This is done using a 'separate' module that 
creates a duplicate of the entity. The original entity then uses a 'signal' module that uses the variable 'line1size' as the signal value to release an entity (representing a can pallet) from one of hold modules that represents the inventory for a specific type of product in the 'inventory' sub-model. The duplicate entity uses a similar 'signal' module to release a lid pallet from the inventory. The value of 'line1size' denotes the size of product to be produced by line 1 during that shift. The decision regarding what to produce in the production lines each shift is done in 'control logic sub-model. The released can and lid pallets are sent to depalletizers. The depalletizers are modeled using 'process' modules followed by a 'separate' module where 99 duplicate entities are created form each 'pallet' entity. The depalletizer converts the pallets into individual cans and lids and sends it to the filling machine through a conveyor. The cans are sent the filler where they are filled with paint. The color of the paint is decided by the 'control logic' submodel. The filled cans are sent to the 'lid stamper' module through the Filler1 Conveyor. The processing time in every machine is represented as a function of the size of the product. Therefore a large sized can will take more time to process than a medium sized can. For example, the processing or 'delay' time in the filling machine is expressed as 'Fillmc1time(Line1Size)' where Fillmc1time is a variable with three rows. If line1size is equal to 1 (which denotes large size) then the value in the first row of the variable will be used. 'Line1Size' value of 2 and 3 represent medium and small sizes respectively. The filled cans and lids are received at the lid-stamper before which a 'match' module is used to group an individual can and lid together. The entity that now represents a can with a lid on it is washed and then sent to a palletizer which a 'batch' module followed by a 'process' module. Once a pallet of finished product is produced, it is sent to the 
appropriate finished goods inventory where its presence is tracked until it is shipped. The nine different types of products are held using nine different 'hold modules' until the 'inventory' sub-model receives a shipping order and uses a 'signal' module to release the products. The releases pallets are then disposed using a 'dispose' module. Once the depalletizers are empty, a signal is sent for the next pallet of cans and lids. The resources in this submodel include the machines and the workers. The number of workers in the four production lines is given by user using a variable named ' $w$ '. This variable has four values in it indicating the number of workers working in production lines 1 through 4 . The machines are modeled using the 'process' module with a 'seize-delay-release' option. The list of various machines and conveyors and the related parameters are listed below.

\begin{tabular}{|c|c|c|}
\hline Machine & Operation & HP \\
\hline Depalletizing Machine1 & Depalletize can pallets & 5 \\
\hline Depalletizing Machine2 & Depalletize lid pallets & 5 \\
\hline Filling Machine & Fill cans with paint & 10 \\
\hline Stamping Machine & Close filled cans with lids & 3 \\
\hline Washing Machine & Wash cans before its packed & 5 \\
\hline Packing Machine & Group cans and pack them with cardboard & 5 \\
\hline Palletizing Machine & Palletize finished cans & \\
\hline
\end{tabular}

Table 3.1 Line 1 Machines 


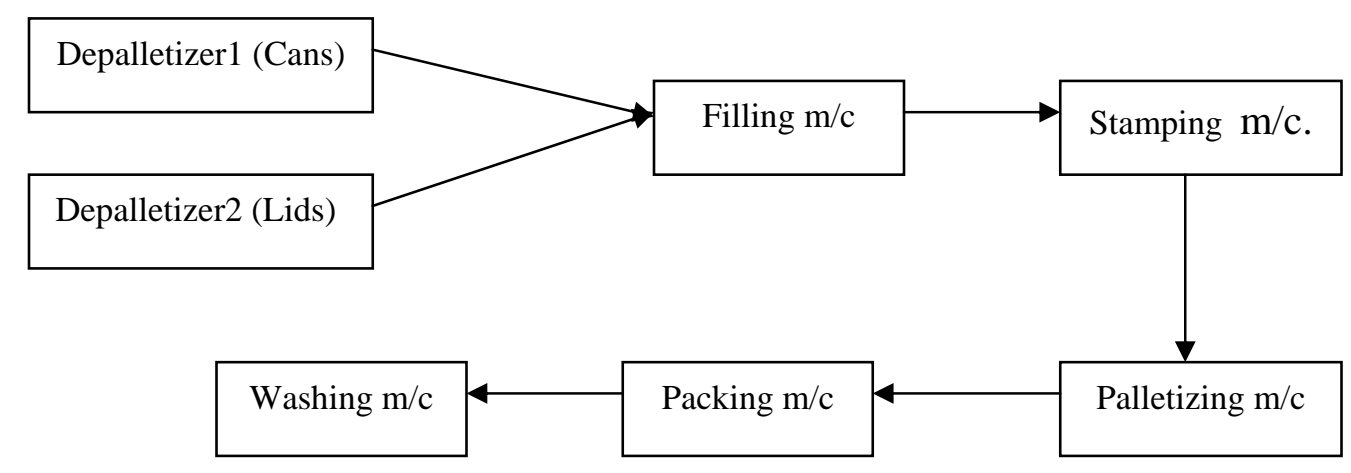

Figure 3.2 Block Diagram of Line 1

\begin{tabular}{|c|c|c|c|c|}
\hline Conveyor Name & Start Station & End Station & Length(ft.) & $\begin{array}{c}\text { Speed } \\
\text { (ft/min) }\end{array}$ \\
\hline Depalletizer1 Conveyor & Depalletizer & Filler1 & 25 & 50 \\
\hline Filler1 Conveyor & Filler1 & Lid Stamper & 15 & 50 \\
\hline Depalletizer2 Conveyor & Depalletizer2 & Lid Stamper & 25 & 50 \\
\hline LidStamper Conveyor & Lid Stamper & Washing & 15 & 50 \\
\hline Prewash Conveyor & Prewash & Packing & 15 & 50 \\
\hline Packing Conveyor & Packing & Palletizer1 & 15 & 50 \\
\hline
\end{tabular}

Table 3.2 Line 1 Conveyors

\subsection{Production Line 2}

This line is contained in the sub-model named steel cans production. This line produces large and medium sized cans. Large size denotes a typical 1-gallon can of dimensions 6-5/8" x 7-1/2", Medium can denotes a typical 1/2 gallon can of dimensions 57/16" x 5-7/8". The line begins with a deep drawing machine which punches blanks from tinplate sheets. Tin plate sheets are obtained in the form of coils and fed to this machine. Using a bodymaker machine, successive drawing is done next on the shallow cups 
initially created. This operation gets the shallow can produced by the deep drawing machine to its final dimension. The edges of the cans are trimmed using a trimming machine. From the trimmer, the cans are sent to a printer where a printed design on the outer surface. The dried can is sent to a beading machine which makes creases that increase resistance to circumferential collapse. After this operation, the cans are palletized and sent to a storage area where it becomes a part of Work In Process Inventory. The machines and conveyors in Line 2 are explained in the following tables.

\begin{tabular}{|c|c|c|}
\hline Machine & Operation & HP \\
\hline Cupper Machine2 & Make shallow cups using deep drawing & 75 \\
\hline podymakess Machine2 & Further draw the cups to the required \\
dimensions & 60 \\
\hline Trimming Machine2 & Trim the cans & 10 \\
\hline Printing Machine2 & Print design on outer can surface & 7.5 \\
\hline Beading Machine2 & Makes creases on the cans & 10 \\
\hline Palletizing Machine2 & Group cans & 5 \\
\hline
\end{tabular}

Table 3.3 Line 2 Machines 


\begin{tabular}{|c|c|c|c|c|}
\hline Conveyor Name & Start Station & End Station & $\begin{array}{c}\text { Length } \\
(\mathrm{ft} .)\end{array}$ & $\begin{array}{c}\text { Speed } \\
(\mathrm{ft} / \mathrm{min})\end{array}$ \\
\hline Cupper Conveyor2 & Cupper2 & Bodymaker2 & 15 & 50 \\
\hline Bodymaker Conveyor2 & Bodymaker2 & Trimmer2 & 15 & 50 \\
\hline Trimmer Conveyor2 & Trimmer2 & Printer2 & 15 & 50 \\
\hline Printer Conveyor2 & Printer2 & Beader2 & 15 & 50 \\
\hline Beader Conveyor2 & Beader2 & Palletizer2 & 15 & 50 \\
\hline
\end{tabular}

Table 3.4 Line 2 Conveyors

It should be noted that the load factor of the machines are different when processing different sizes of cans because lower effort is required by motors to process medium sized cans. This has been taken into consideration. A load factor of $85 \%$ is used for large cans and $65 \%$ is used for medium cans. These values have been arrived at following discussion with various equipment manufacturers and information obtained during energy audits performed by the Industrial Assessment Center. But they can be easily changed by the user to represent a specific plant. A load factor of $10 \%$ has been used for machines in the idle state. The machines in this line are connected by conveyors. All conveyors are accumulating type conveyors assumed to be operating at a load factor of $85 \%$ when there are products on them and at $50 \%$ load when there are no products on them.

The considerable setup time incurred when changing the production run from large cans to medium cans and vice-versa has been considered. Typical setup times have 
been obtained after discussing with manufacturers. The machines are considered to have a load factor of $10 \%$ when being setup.

Entities representing individual large or medium size cans are produced in this sub-model. The entities are disposed if the control-logic sub-model decides not to operated this line. Otherwise the entities proceed to Cupper machine where the processing time is decided based on the size of the cans that is decided by the control logic sub-model. Every machine in this line has different processing times based on the product. Assigning the processing time for a product based on its size is done in the same way that its done in Line 1 sub-model. Once the can goes through the bodymaker, trimmer, printer and beader it is sent to a 'batch' module. Once a hundred pieces are produced, a pallet is produced by the 'batch' module and then sent to a 'process module' (palletizing machine). The pallets are then sent to the WIP inventory. The time at which the pallet enters the inventory is assigned to the pallet as an attribute so that it can be eventually used to calculate the time spent in the inventory. The WIP inventory consists of 'hold' modules that release the pallets to the depalletizer in Line1 once it receives a signal.

\subsection{Production Line 3}

This line is contained in the sub-model named Line 3. The operations in Line 3 are the same as Line 2 except for the fact that it produces only small cans. It operates at a load factor of $85 \%$ when active and $10 \%$ when idle. 


\begin{tabular}{|c|c|c|}
\hline Machine & Operation & HP \\
\hline Cupper Machine3 & Make shallow cups using deep \\
& drawing process & 40 \\
\hline Bodymaker Machine3 & Further draw the cups to the \\
required dimensions & 10 \\
\hline Trimming Machine3 & Trim the cans & 5 \\
\hline Printing Machine3 & Print design on outer can surface & 7.5 \\
\hline Beading Machine3 & Makes creases on the can & 5 \\
\hline Palletizing Machine3 & Group cans & \\
\hline
\end{tabular}

Table 3.5 Line 3 Machines

\begin{tabular}{|c|c|c|c|c|}
\hline Conveyor Name & Start Station & End Station & Length(ft.) & Speed(ft./min) \\
\hline Cupper Conveyor3 & Cupper3 & Bodymaker3 & 15 & 50 \\
\hline Bodymaker Conveyor3 & Bodymaker3 & Trimmer3 & 15 & 50 \\
\hline Trimmer Conveyor3 & Trimmer3 & Printer3 & 15 & 50 \\
\hline Printer Conveyor3 & Printer3 & Beader3 & 15 & 50 \\
\hline Beader Conveyor3 & Beader3 & Palletizer3 & 15 & 50 \\
\hline
\end{tabular}

Table 3.6 Line 3 Conveyors

The sub-model Line 3 is very similar to Line 2 except for the fact that Line 3 produces only small sized products. There is only one hold module for the WIP inventory because there is only one type of product to be tracked. Entities representing individual cans are produced in this sub-model. The entities are disposed if the control-logic submodel decides not to operated this line. If it is decided that Line 3 will be operated, the 
entities proceed to the cupper machine. Once the entity goes through bodymaking, trimming, printing and beading, it is sent to a palletizer. The palletizer is a 'batch module' followed by a 'process' module. A pallet entity is created and sent to the WIP inventory once hundred pieces are produced. Its time in the inventory is tracked to calculate the carrying cost.

\subsection{Production Line 4}

This line is contained in the sub-model named Line 4 . This line has the capability of producing corresponding slip-lids for the cans produced in Line 3 and Line 4. Since there are three different sizes of lids, there will be a considerable setup time involved when changing the production from one size to another.

The line starts with a press that produces the lids from uncoiled tinplate. The lids are then transferred to a trimmer where the lids are trimmed to the exact dimensions. The trimmed cans go to a printer which prints the required design on the lids. The printed lids are palletized into groups of 100 and stored as a part of Work In Process inventory. The load factors of the machines while producing large, medium and small lids are 85\%, 75\% and $60 \%$ respectively. The load factor is $10 \%$ when the machines are idle. The machines in this line are connected by conveyors. All conveyors are accumulating type conveyors assumed to be operating at a load factor of $85 \%$ when there are products on them and at $50 \%$ load when there are no products on them.

Sub-model 4 is very similar to Line 2 . The decision to operate the line and the size of the lids produced in this line is done in the 'Control Logic' sub-model. Entities representing individual lids are produced in this sub-model. The entities are disposed if the control logic sub-model decides not to operated this line. If the line is operated in that 
shift, the entities proceed to the press and then to the trimming and printing machines before being palletized. The processing time on the machines will depend on the type of the product produced. The pallets are finally sent to one of the appropriate WIP inventory. The inventory is a set of three 'hold' modules used to hold the pallets before they are released to the depalletizer in line 1 . The time that the lid pallets are in the WIP inventory is tracked to calculate carrying cost.

\begin{tabular}{|c|c|c|}
\hline Machine & Operation & HP \\
\hline Press Machine4 & Make shallow lids & 40 \\
\hline Trimming Machine4 & Trim the lids to the required \\
dimensions & 10 \\
\hline Printing Machine4 & Print designs on the lids & 5 \\
\hline Palletizing Machine4 & Palletize lids for storing in WIP & 5 \\
\hline
\end{tabular}

Table 3.7 Line 4 Machines

\begin{tabular}{|c|c|c|c|c|}
\hline Conveyor & Start Station & End Station & $\begin{array}{c}\text { Length } \\
\text { (ft.) }\end{array}$ & $\begin{array}{c}\text { Speed } \\
\text { (ft./min) }\end{array}$ \\
\hline Press Conveyor4 & Press & Trimmer4 & 15 & 50 \\
\hline Trimming Conveyor4 & Trimmer4 & Printer4 & 15 & 50 \\
\hline Printing Conveyor4 & Printer4 & Palletizer4 & 15 & 50 \\
\hline
\end{tabular}

\section{Table 3.8 Line 4 Conveyors}

\subsection{Costs Involved}

The following costs incurred by the facility are kept track of by the simulation model. The unit costs to be used in calculating the following costs are given by the user 
as input. The user can do it by clicking the 'variable' icon in the Basic Process template and editing the variable that represents the parameter to be changed.

1. Inventory Carrying Cost - This cost is provided by the user and expressed in dollars per pallet per day. There are three different variables (arrays) containing three values each that represent carrying cost in this model. The variables that represent the carrying cost of WIP cans, WIP lids and finished goods are cancarrycost, lidcarrycost and fincarrycost respectively. It is assumed that products of the same size have the same carrying cost regardless of color. Since the number of days that a pallet spends in the inventory is tracked, the carrying cost per day is used to calculate the total carrying cost. This calculation is done using the 'expression' option found in the Advanced Process template. The result obtained from the expression named totalcarrycost is exported to a spreadsheet called costs.xls after every shift.

2. Stockout Cost - Stockout cost is provided by the user in dollars per pallet. The inventory sub-model generates entities that represent incoming orders and compares the size of the orders to the inventory. If a stockout situation arises, the stockout cost per pallet given by the user is multiplied to the number of pallets to calculate the total stockout cost. The number of stockouts is also recorded in the variable called noofstockouts. The stockout cost per pallet given by the user is represented by a variable named stockoutcost. This variable accepts nine different costs for nine different products. The total stockout cost calculated is exported to a spreadsheet named costs.xls after every shift. The day and quantity of stockout is exported to a spreadsheet called stockout.xls. 
3. Labor Cost - The regular labor cost per hour and the premium labor cost per hour for the second shift are stored in the variables called offlaborcost and onlaborcost respectively. The number of workers in the four production lines is stored in the variable called $\mathrm{w}$. The user can change the number of workers and also the labor cost per hour using these variables. When a line is operating, the number of workers in the line is multiplied by the appropriate labor cost and the result is stored in the expression called 'totallaborcost'. The total labor cost calculated is exported to a spreadsheet named costs.xls after every shift.

4. Electric Usage Cost - The on-peak electric usage cost and off-peak electric usage cost are stored in the variables named onusagecost and offusagecost respectively. The usage cost is expressed in $\$ / \mathrm{kWh}$. The energy meter sub-model records both off and on-peak kWh which is multiplied by the kWh cost provided by the user to calculate the total usage cost.

5. Electric Demand Cost - The on-peak demand cost and off-peak demand cost are stored in the variables named ondemandcost and offdemandcost respectively. The demand cost is expressed in $\$ / \mathrm{kW}$. The energy meter sub-model records both off and on-peak $\mathrm{kW}$ which is multiplied by the $\mathrm{kW}$ cost provided by the user to calculate the total demand cost 


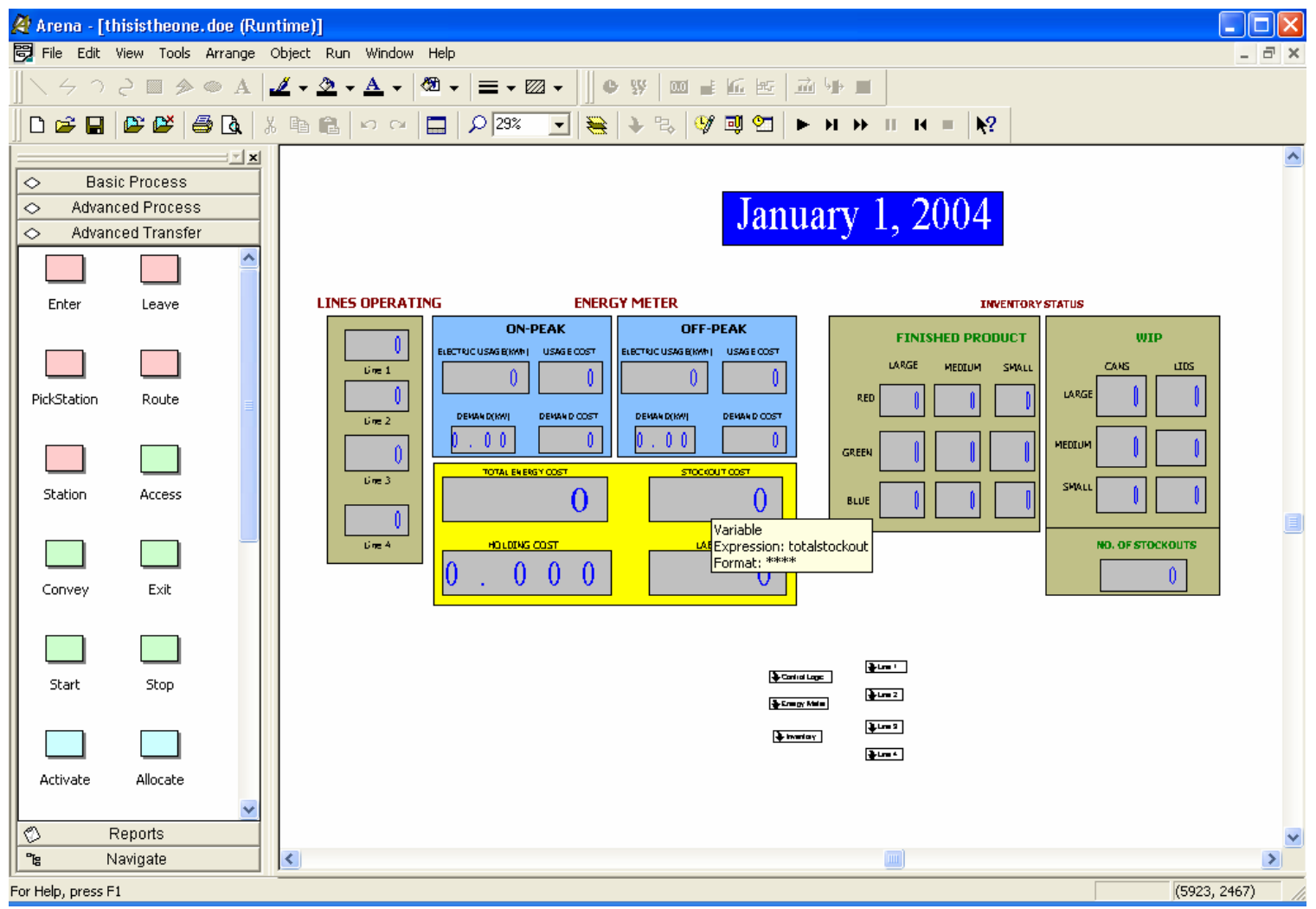

Figure 3.3 Snapshot of the interface showing various costs

\subsection{Inventory Tracking}

All three sizes of cans and lids are considered Work In Process inventory until used in Line1 and sent out as finished product. The simulation model keeps track of three sizes of cans and three sizes of lids at any point of time during the simulation. Similarly, all the nine varieties of finished products ( 3 sizes and 3 colors in each size) are tracked.

Holding the entities representing the products and WIP is done using hold module. The 'signal' to release a specific number of pallets (entities) comes from the logic that simulates incoming orders. This logic is in the 'inventory' sub-model. Inventory carrying cost is calculated for both WIP and finished goods. The time when every pallet enters and leaves the inventory is recorded and the time it was held in the inventory is calculated. Using the unit carrying cost for each kind of product given by the 
user, the total cost of holding the inventory is calculated. A typical holding cost per year of $20 \%$ of the cost of the product is used in this model. This value can be changed by the user if necessary.

The 'inventory' sub-model performs two main functions. It contains the logic that creates orders, signals shipping of entities, monitors stockouts and also contains the modules that writes the required output to spreadsheets. These two logics are independent of each other. The 'shipping' logic creates entities that represent the orders once every 7 days after the build-up period is over. Once the entity is created, it goes through 'assign' modules that assign variables that decide the number of orders, products ordered and size of the orders using probability distributions. The expression 'ordersperweek' is the probability distribution that decides the number of orders. It is a discrete distribution with a $33 \%$ probability of having 1 or 2 or a maximum of 3 orders. The expression 'shipquantitydist' represents the number of pallets ordered. The probability distribution is TRIA $(40,50,60)$. The entity goes to a 'decide' module where the order size is compared against the number of pallets in stock for the product ordered. If there are not enough pallets in stock, the variable 'noofstockout' is increased by one. This variable records the number of stockouts that occur during the simulation. The stockout cost is also calculated at this point. The difference between the size of the order and the pallets in stock is considered as lost sales and the stockout cost is the calculated by multiplying the stockout cost/pallet and the difference in the number of pallets. A 'signal' module is used next to signal to release a specific number of products, which is either the size of the order or the number of pallets that is available readily to ship. The entity then goes through a 'decide' module that checks if all the orders for that week have been shipped. If not, the entity 
goes through the same series of module explained above until the number of orders is equal to what the probability distribution came up with.

A stockout (lost sales) cost of $10 \%$ of the selling price of the product is used in this model. Specific stockout cost for any of the nine types of finished products can be changed by the user if necessary.

Shipping out finished products can be controlled by input from the user. Currently, shipping is set to occur every week and the quantity of orders and type of products are based on probability distributions. The number of stockouts and their corresponding cost are also tracked.

Exporting the required output to spreadsheets are done using 'Read/Write' modules. There are total of eleven 'Read/Write' modules in the inventory sub-model that write data to eleven different spreadsheets. These modules have a 'create' module before them that create entities at different time intervals (every hour or every shift or every day) depending on the type of the output. These modules can be changed by the user to export any kind of data as required.

All the raw material required for producing the cans and lids, and the paint filled in the cans are considered JIT (Just In Time) and their inventories are not considered in this model. This research concentrates only on the cost of WIP and Finished Goods inventory and how they may be affected by a schedule designed to minimize electric usage and demand.

The inventory sub-model creates entities that represent the orders for products based on a probability distribution. The size of the order is compared with the stock in the finished goods inventory. The entity sends a signal to 'ship' or dispose the required 
number of pallets from the inventory if there is enough stock in the inventory. Otherwise a stockout is recorded and the cost of lost sales is calculated. The inventory sub-model also contains the logic to export the output to spreadsheets.

\subsection{Labor}

The labor cost incurred to operate the production lines is also taken into consideration. Should there arise a necessity to operate the facility during off-peak hours, a premium will be added to the regular labor cost. The labor cost per hour is provided by the user and can be changed easily as necessary. The number of personnel required to operate each line is given by the user and can be changed if necessary. It is assumed that all the workers are available as and when required.

Tracking labor cost is also done in the inventory sub-model. An entity is created at the beginning of every shift and it checks the results from the 'control logic' sub-model for the production lines that are being operated. The entity goes to a 'decide' module next where it checks if the current shift is off-peak or on-peak. This step is to make sure that it uses the correct labor cost. Using the number of workers and labor cost provided by the user, the entity then calculates the labor cost and finally disposes itself.

\subsection{Energy Usage Monitoring}

Different costs can be assigned for off-peak and on-peak electricity and the user can also designate the off-peak and on-peak hours using the applicable rate schedule. Changing the peak hours can be done by using the variables 'onstart' and 'onstop' denoting the start and stop of on-peak hours respectively. The model is designed in such a way that it can handle a tariff with different on-peak and off-peak rates for both electric usage and demand. 
The simulation model mimics the way a real kilowatt meter installed by the utility records the power used by a facility. Different electric meters have different sampling rates. A typical sampling rate observed in many commercially available electric meters is about 32 samples per second. Although this sampling rate is used in the model, it can be changed by the user to incorporate the sampling rate of any particular meter. The electric demand updates itself every 15 minutes just like a real electric meter. Electric usage cost is also calculated using the unit costs provided by the user.

Entities are created in this sub-model at a rate comparable to the sampling rate of an electric meter. The user can change this rate by editing the variable called samplesperminute. The entity first checks if it is the end of a month using a 'decide' module. If yes, it records the highest on and off peak $\mathrm{kW}$, adds the current month's demand charge to the sum of the demand charges of the previous months (represented by the variable 'cumuldemandcharge') and then proceeds to the next 'assign' module which records instantaneous $\mathrm{kW}$ (represented by the variable 'reading'). If it was not the end of a month, the entity would have directly come to the module that records instantaneous $\mathrm{kW}$. Recording instantaneous $\mathrm{kW}$ involves a large number of expressions that check the current status of the machines and conveyors to determine the load factor. For example, to calculate the instantaneous $\mathrm{kW}$ of Line 1, denoted by L1Total, the model will evaluate two other expression $\mathrm{L} 1 \mathrm{M}$ and $\mathrm{L1C}$ which are the instantaneous $\mathrm{kW}$ of the all the machines and all the conveyors in Line 1 respectively. L1M is defined as an expression which is the sum of the product of the $\mathrm{kW}$ of the machine, the load factor and the status variable. The status is 0 if the machines are idle and 1 if they are operating. $\mathrm{L} 1 \mathrm{C}$ is also definitely similarly for the conveyors. The sum of L1M and L1C gives L1Total. The 
same method is used for all the four production lines to calculate the instantaneous $\mathrm{kW}$. The entity then checks if it is the end of a 15 minute interval. If so the average 15 minute demand is calculated and compared to the previously recorded highest average demand. If the new average $\mathrm{kW}$ is higher then it is recorded. Then the entity goes to a 'decide' module where it checks if it is on-peak or off-peak at present. Depending on the result of this check, the entity takes one of two paths - on-peak or off-peak. All the calculations from this point are done separately for on-peak and off-peak periods. This is to make sure the correct costs are used depending on the shift. The entity proceeds to calculate usage costand then gets disposed.

\subsection{Conclusion}

The simulation model has been developed to help see the effects of a demand reduction strategy on the overall cost. Though the parameters used here are generic and realistic, they can be changed to represent any particular situation. A reasonable level of complexity has been maintained so that it does not interfere with the objective of the research. 


\section{Chapter 4}

\section{System Implementation}

The manufacturing setup used here belongs to a type of operation which is not uncommon particularly among the mid and small scale industries that the IAC at WVU has performed energy audits. This is a setup in which the plant manager has a few production lines to produce a variety of products and the number and size of the orders vary a lot. The size and the number of orders are given in the form of probability distributions. The plant manager uses all the resources available to avoid stockouts. Since he does not really know what the next order would be, he cannot precisely decide whether a demand reduction strategy would affect his profits or not. His selection of the lines to be operated on a particular shift is based on some logic that he has learnt from his experience and traditionally how the general demand for various products have been. The scenarios analyzed here are based on the assumption that, in the event of an order, there is a $50 \%$ chance of getting an order for small sized product, $25 \%$ chance for a medium sized product and a $25 \%$ chance for a small sized product. This is the reason there is a dedicated line for producing small sized cans.

\subsection{Scenarios analyzed}

Three scenarios have been chosen to analyze the effect of measures intended to minimize electric demand on the overall cost. The difference between these scenarios is the number of production lines being run during the first shift when peak demand rates apply. The maximum number of production lines operating at the same time in the first, second and third scenarios are four, three and two respectively. All other parameters and the logic used to decide which line to operate remain the same. 


\subsection{Scheduling and Control Logic}

The Control Logic sub-model does the function of deciding which lines are to be operated in any shift and what type of products are to be produced. At the beginning of every shift, depending on the input from the user regarding how lines can be operated, an entity is created using a 'create' module that goes to a 'decide' module where it's routed to the applicable logic. 'Separate' modules are used to create as many duplicates as required of this entity to control the operations of the production. There are four more sub-models within the 'control logic' sub-model. They are named Control Line 1, Control Line 2, Control Line 3 and Control Line 4. Depending on the number of lines the user wants to operate, the entities created will be sent to two or more of these four sub-models. The 'control line 1' sub-model checks the availability of empty cans and lids to operate line 1 . If there are enough WIP available the variable called 'line1production' is set to a value of 1 which indicates that the line is ready for operation. Then the entity disposes itself. If there is not enough WIP, the entity goes to one of the other three submodels to select which one of those can be operated. The reason behind this logic is that line 1 is given the highest preference by the user so that he can get as many finished products out as possible. The other sub-models simply check the maximum number of lines that can be operated, how many other lines have been already selected and set the value of the variables ‘line2production', ‘line3production', ‘line4production’ to either 1 or 0 accordingly. The logic to choose, at the beginning of each shift, the lines and the size of the product to be produced in that line is as follows:

1. The filling line (Line 1) is given the first preference because the objective is to get the maximum possible number of finished products out. The WIP inventory is checked to see if there are enough lids and cans of a particular size 
to run the filling line for one full shift. The order of checking WIP inventories of the three different sizes is based on the demand for the different sizes of products. This demand is represented by a Discrete distribution. All the three colors considered in this model (Red, Blue and Green) have equal chances (33.3\%) of being assigned.

2. Line 2, which produces large and medium sized cans, is operated based on the maximum number of lines allowed. There is an equal chance for either size to be produced since the demand for the finished products of both the sizes are equal.

3. Line 3 produces only small cans and the only decision to be made is whether it should be operated or not. This decision is made depending on how many lines can be operated at the same time.

4. Line 4 produces all three sizes of lids and the size to be produced is determined based on the number of cans in the WIP inventory. The size with the highest difference between the number of WIP cans and WIP lids is chosen to maintain a balance between the three different sizes of WIP inventories. This line primarily makes sure that there are enough lids corresponding to the number of cans so that Line 1 can be operated to the maximum possible extent. Production of lids takes much less time than the production of cans due to the nature of the process.

\subsection{Scenario 1 Logic (Maximum of 4 production lines at any time)}

In this scenario, the WIP inventory is first checked at the start of a shift for availability of cans and lids of any of the three sizes. The user decides what the minimum 
number of components required based on his experience. Since the small sized finished product is in higher demand than the other two sizes, it is given more importance while checking the WIP inventory. If there is enough components (cans and lids) of any of the three sizes, line 1 is operated during the shift. The color of paint filled in the cans is then assigned to the product. All the three colors considered in this model (Red, Blue and Green) have equal chances (33.3\%) of being assigned. This is represented in the model as a discrete distribution, which can be changed if required by the user. All the other three lines are also operated and the selection is done according to the rules mentioned in the previous section. The set-up time required during the beginning of each shift, especially when the product changes, is provided by the user in the form of a probability distribution. The distributions representing the set-up time for line 1,2 and 4 are listed as expressions called line1changetime, line2changetime and line4changetime respectively. It is assumed that line 3 has a negligible set-up time since it produces only one type of product. The user can change these probability distributions if necessary.

\subsection{Scenario 2 logic (Maximum of 3 production lines at any time)}

In this scenario, the WIP inventory is first checked at the start of a shift for availability of cans and lids of any of the three sizes. Since the small sized finished product is in higher demand than the other two sizes, it is given more importance while checking the WIP inventory. If there is enough components (cans and lids) of any of the three sizes, line 1 is operated during the shift. If line 1 is not operated due to unavailability of components, lines 2, 3 and 4 are operated according to the rules mentioned in the previous section. 
If line 1 is operated, the selection of two lines out of the three available lines is done in the following way. If the number of lids of all the three sizes is higher than the number of corresponding cans, the lid line (line 4) is not operated - lines 2 and 3 are operated. Otherwise line 4 and either line 2 or 3 are operated. Lines 2 and 3 have equal chance of being operated.

\subsection{Scenario 3 logic (Maximum of 2 production lines at any time)}

Since the combined $\mathrm{kW}$ of line 3 and 4 is a little less than the combined $\mathrm{kW}$ of line 1 and 2, they will be operated during the peak shift. Lines 1 and 2 will be operated in the second shift. If there are not enough components to operate line 1, only line 2 will be operated during the second shift. Selection of the size and color in any of the lines will be according to the rules explained in the previous section.

\subsection{Input values provided by the user}

The variables for which the user can provide the values are as follows. Although they can be changed if required, their current values as used in this model are listed below. Again, it may be noted that these values can vary significantly from one facility to the other but care has been taken to use typical values seen in the industry. Most of these values were the result of discussions with plant managers, vendors, original equipment manufacturers and data available through the IAC audits at WVU. The electric rates closely resemble Central Vermont Public Service’s Rate 4 - Primary Service, the most widely used rate schedule among the manufacturers in Vermont.

1. Peak electric usage cost -8 cents/kWh

2. Off-peak electric usage cost -5 cents/kWh

3. Peak electric demand cost - $\$ 12 /(\mathrm{kW}$.month) 
4. Off-peak electric demand cost - \$0/(kW.month)

5. Regular labor cost $-\$ 8 /$ hour

6. Off-peak labor - $\$ 10 /$ hour

7. Carrying cost for cans

\begin{tabular}{|c|c|}
\hline Size & \$/pallet/day \\
\hline Large & 0.047 \\
\hline Medium & 0.039 \\
\hline Small & 0.021 \\
\hline
\end{tabular}

8. Carrying cost for lids

\begin{tabular}{|l|c|}
\hline Size & \$/pallet/day \\
\hline Large & 0.011 \\
\hline Medium & 0.011 \\
\hline Small & 0.010 \\
\hline
\end{tabular}

9. Carrying cost for finished product

\begin{tabular}{|c|c|}
\hline Size & \$/pallet/day \\
\hline Large & 0.427 \\
\hline Medium & 0.288 \\
\hline Small & 0.173 \\
\hline
\end{tabular}


10. Stock-out cost (lost sales)

\begin{tabular}{|l|c|}
\hline Size & \$/pallet \\
\hline Large & 104 \\
\hline Medium & 70 \\
\hline Small & 42 \\
\hline
\end{tabular}

The model was run with the input values shown above and all the three scenarios mentioned earlier in this section were analyzed. A build up time of 15 days was used. Build-up time in this context means how many days of stock would be allowed to build up before they receive their first shipping order. If this build-up time is short, then the company will incur a higher stockout cost but the carrying cost may be lower due to products being shipped early. To enable better comparison, it was chosen to run the simulation for 270 days (approx. 9 months). Since there was not much change in the output with each replication, the results used for analysis were the average of two replications.

\subsection{Analysis of the results obtained}

The first set of outputs to be analyzed below is the one obtained by running the simulation model with a build-up period of 15 days. The following pie charts show how the various costs changed when a maximum of two, three and four production lines were operated at any time. 


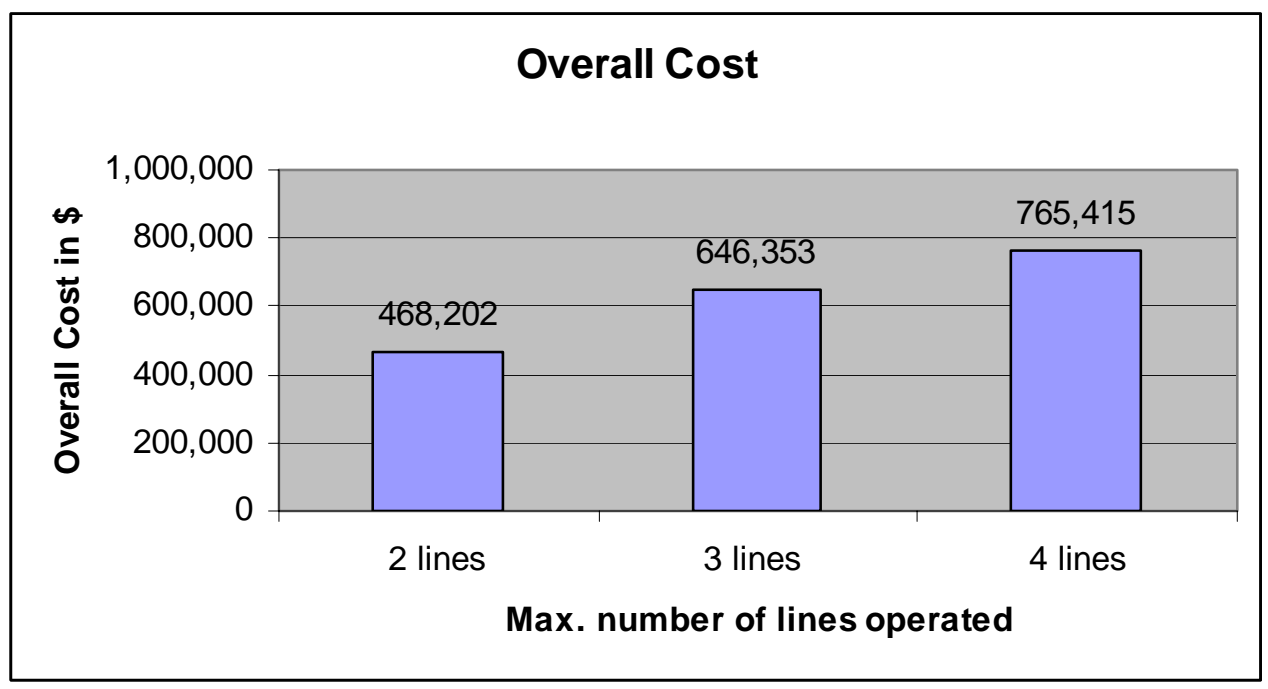

Figure 4.1 Total cost in different scenarios

It can be noticed that the cost increases with the number of lines being operated at the same time. But the difference in the cost between operating two and three lines is less than that of three and four lines. This is due to the limitations in operating the finishing line. According to the logic, it can be operated only if there is enough lids and cans in the WIP to run it for one full shift. Therefore, the operation ends up running only 3 lines though the finishing line is allowed to operate. Though the overall cost is a good piece of information that can be used by management, it should be analyzed in conjunction with the number of pallets of various finished products and WIP in the inventory. The profit margin of the products will finally decide the most economical option for the user. Other options such as the ability to sell empty cans and lids to other manufacturers or subcontract one or more products are options that can change the perspective of the user towards selecting the best scenario. 


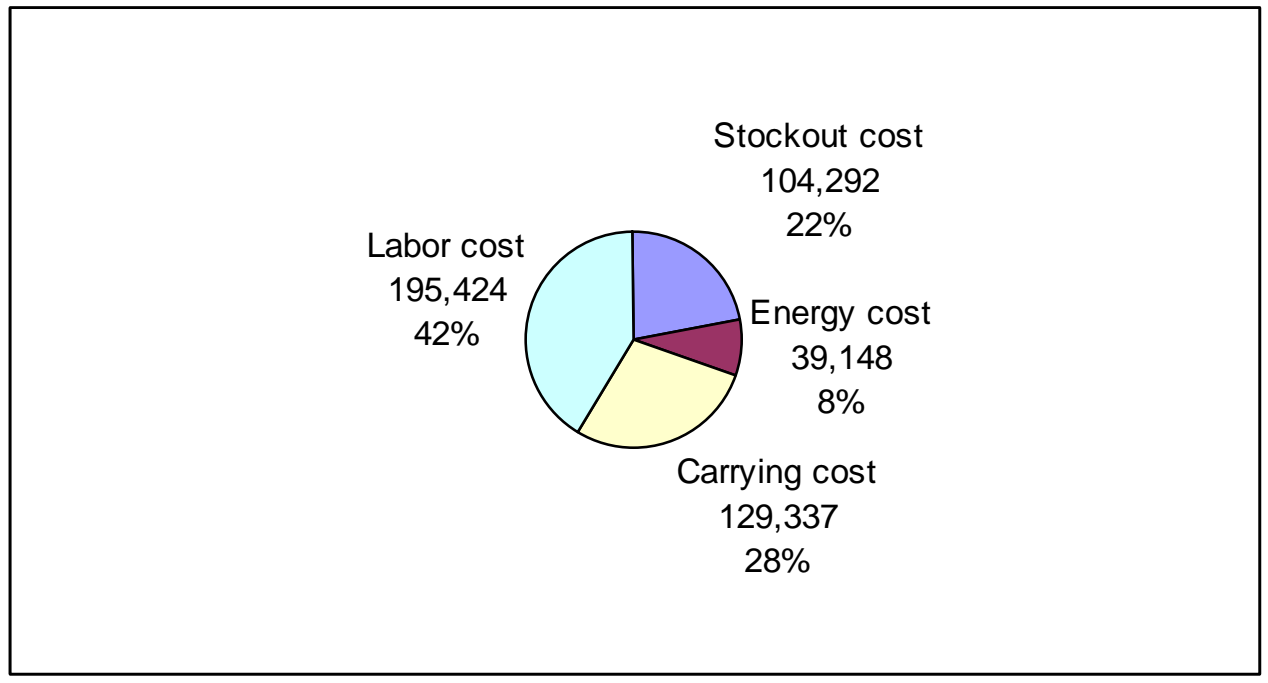

Figure 4.2 Distribution of costs with a maximum of two lines in operation

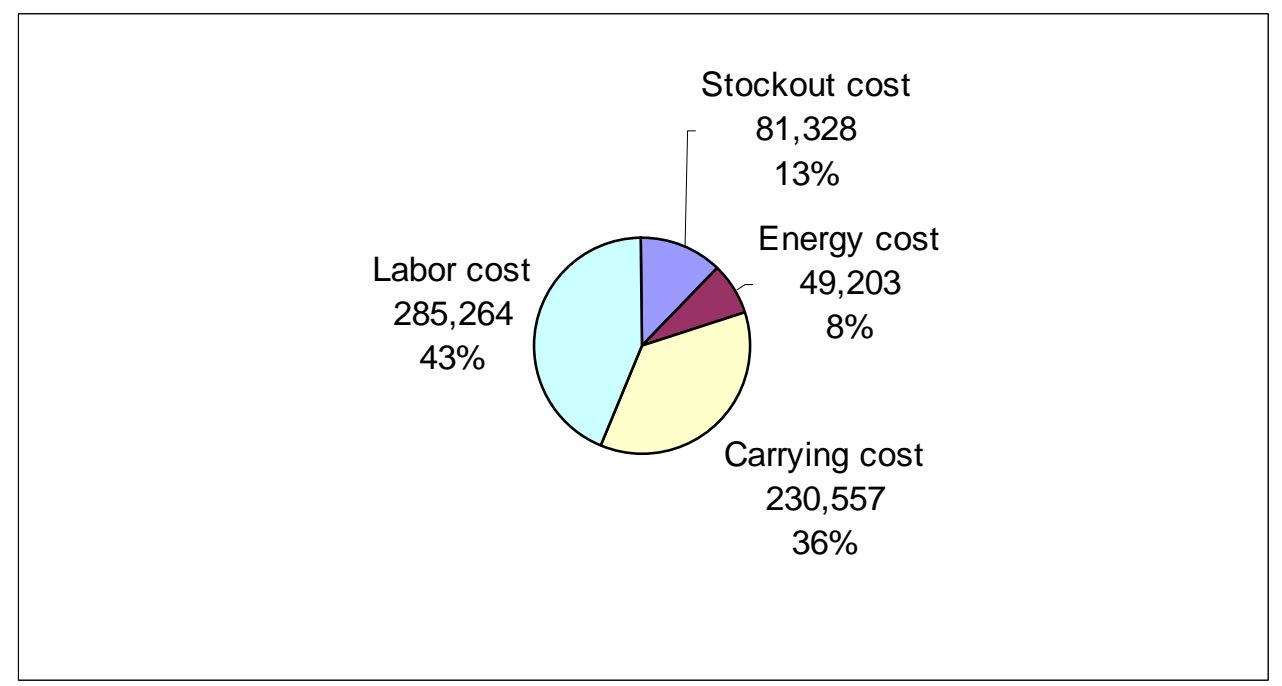

Figure 4.3 Distribution of costs with a maximum of three lines in operation 


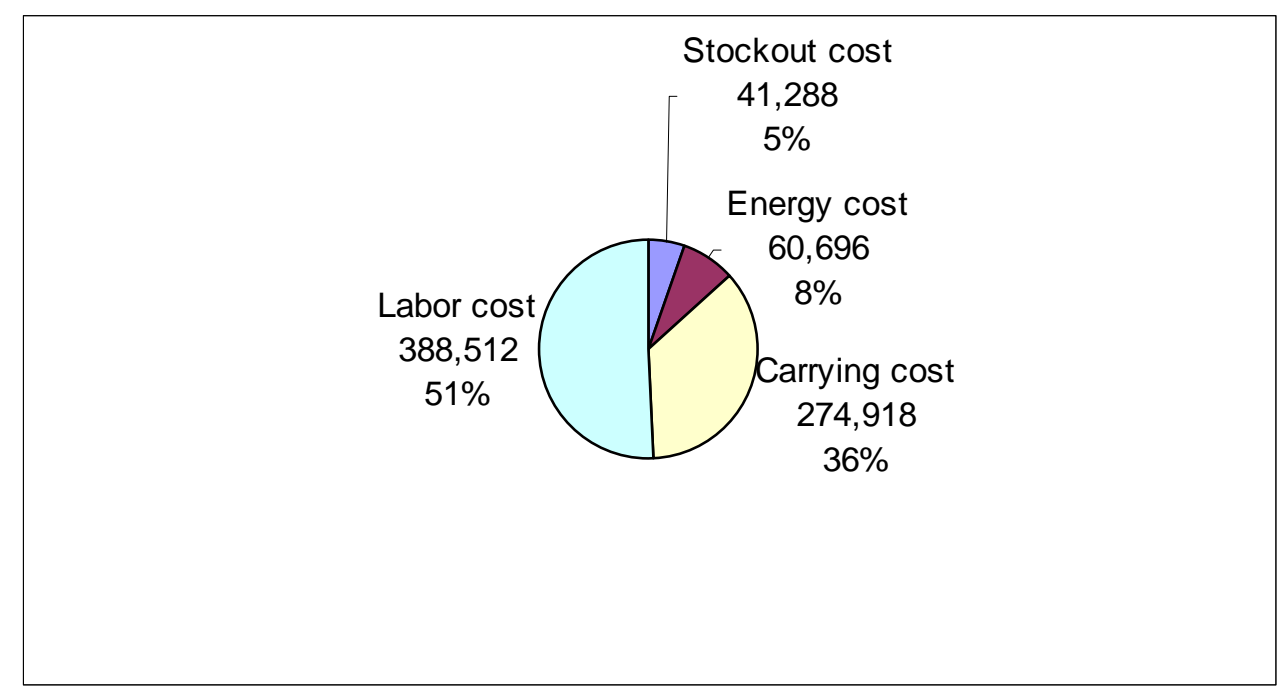

\section{Figure 4.4 Distribution of costs with a maximum of four lines in operation}

Figures 4.3, 4.4 and 4.5 show the distribution of various costs in the operation. It should be noted that the cost of raw material and overheads which are typically a big share of the overall cost in the can-making industry is not being considered in this model. This may make the labor and carrying cost appear overstated. It can be seen that the size of the stockout component reduces while the labor and carrying cost components get bigger with more lines being operated. These results agree with the reasoning that when more lines are operated, there are more products and more worker-hours that lead to a lower number of stockouts. All the graphs shown in this section are those obtained from the excel spreadsheets produced by the simulation program. 


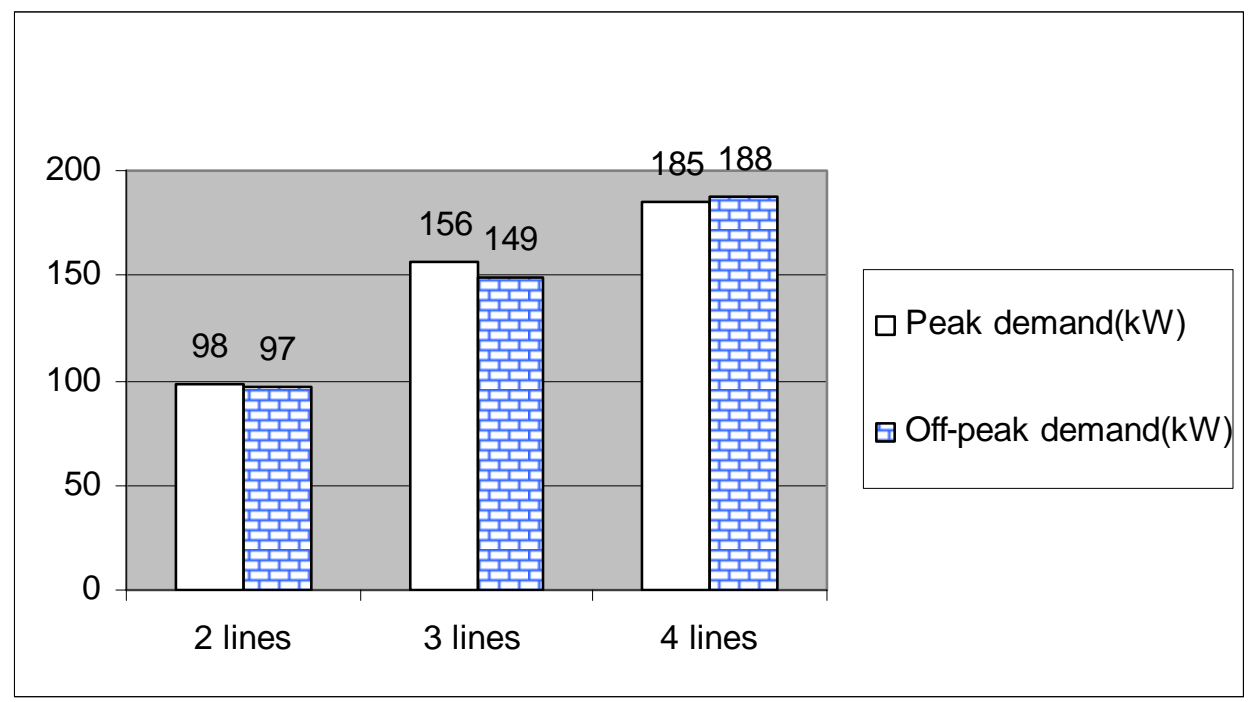

Figure 4.5 Comparison of peak and off-peak demand

The manufacturing facility simulated is a 2-shift, 7 days per week operation. The first 8 hours are designated as peak hours which mean higher electric cost and lower labor cost associated with them. The second 8 hour shift is off-peak with lower electric cost but higher labor cost. Since the maximum number of lines operated in the two shifts is the same in any scenario, the on and off-peak demands recorded by the energy metering sub-model are very close to each other. The difference in peak $\mathrm{kW}$ between different scenarios is due to the limit imposed on the maximum number of lines that can be operated. The difference in $\mathrm{kW}$ compares with the total $\mathrm{kW}$ of the production lines not operated after factoring in the load factor. 


\begin{tabular}{|c|c|c|c|}
\hline & 2 lines max. & 3 lines max. & 4 lines max. \\
\hline & \multicolumn{3}{|c|}{ WIP Cans Inventory } \\
\hline Large & 278 & 441 & 781 \\
\hline Medium & 4,375 & 5,444 & 8,279 \\
\hline Small & 6,199 & 8,042 & 12,353 \\
\hline & 110 & 449 & 441 \\
\hline Large & 4,201 & 5,471 & 7,934 \\
\hline Medium & 5,993 & 8,052 & 12,000 \\
\hline Small & 107 & Finished Products Inventory \\
\hline & 1,359 & 416 & 429 \\
\hline Large & 3,376 & 2,431 & 2,656 \\
\hline Medium & \multicolumn{3}{|c|}{ Electric Usage (kWh) } \\
\hline Small & 159,813 & 244,538 & 314,549 \\
\hline & 166,405 & 252,698 & 313,247 \\
\hline Peak Usage & \multicolumn{3}{|c|}{} \\
\hline Off-peak usage & \multicolumn{3}{|c|}{} \\
\hline
\end{tabular}

Table 4.1 Results (15 days build-up period)

Table 4.1 shows how many pallets of both WIP and finished good were produced in the simulation. The electric usage in the peak and off-peak hours are also shown as recorded by the energy meter sub-model. The number of finished products and WIP cans and lids increase with the increase in the number of lines operated. The size of the inventory may be beneficial or not depending on whether any use can be made of the parts in the WIP inventory. The results are open-ended and the user has to decide the best way to go based on his knowledge and experience.

\subsection{Conclusion}

Like in any case, though there are some similarities, every operation is unique in its own way. There are infinite ways that this data can be used to compare different scenarios. And the outputs can be used to make certain decisions, which can change the advantage of one scenario over the other. The final decision may sometimes be based on decisions that are other than financial. Some businesses consider keeping customers 
happy is more important than short-term profits. In that case the number of stockouts can matter more than the stockout cost. Logistics can play a more important role. For example, if the user is close enough to other sources from where he can sub-contract or outsource his requirements in case of an order, he does not have to worry about stockout a lot and concentrate on reducing other costs. Also, the decision will depend on how much the budget and target profit is. He can simply choose to avoid risk, spend less and make less money. The user may also negotiate with a utility for a rate schedule that would match their loads and still costs less - an option which is adopted by a lot of big industrial and commercial customers these days. In that case, it would help to modify this model according to the intended strategies and analyze the output to see if that may a right decision. Even if there is not much randomness, it is still a good tool that can give the plant manager some useful numbers to work with before taking decision that can affect the profitability. Though this model does not use real-world data, the results show how the user can use this approach to make the right decisions when it comes to reducing cost through electric demand reduction. 


\section{Chapter 5}

\section{Sensitivity Analysis}

One of the primary objectives of this effort is to be able to identify the factors that affect the overall cost and how they affect it. The effect of different factors on the cost of the product is analyzed here. The scenario with a build-up period of 30 days is chosen and the analysis is performed for the three cases with a maximum of one, two and three lines being operated simultaneously.

\subsection{Factors to be considered}

It will be discussed how a change in the value of the following parameters affect the overall cost.

1. Peak usage cost

2. Peak demand cost

3. Off-peak usage cost

4. Off-peak demand cost

5. Carrying cost

6. Stockout cost

7. Size of orders

8. Labor cost

Each of the parameters above was changed by $25 \%$ while keeping all the other parameters unchanged and the effect on the overall cost was recorded.

\subsection{Effect of various factors}

The following results were obtained when the each of the parameters mentioned above were reduced or increased by $25 \%$ with a maximum of 2 lines in operation. It can be seen that the carrying cost and labor cost have a pronounced effect on the overall cost more than any other factors. 


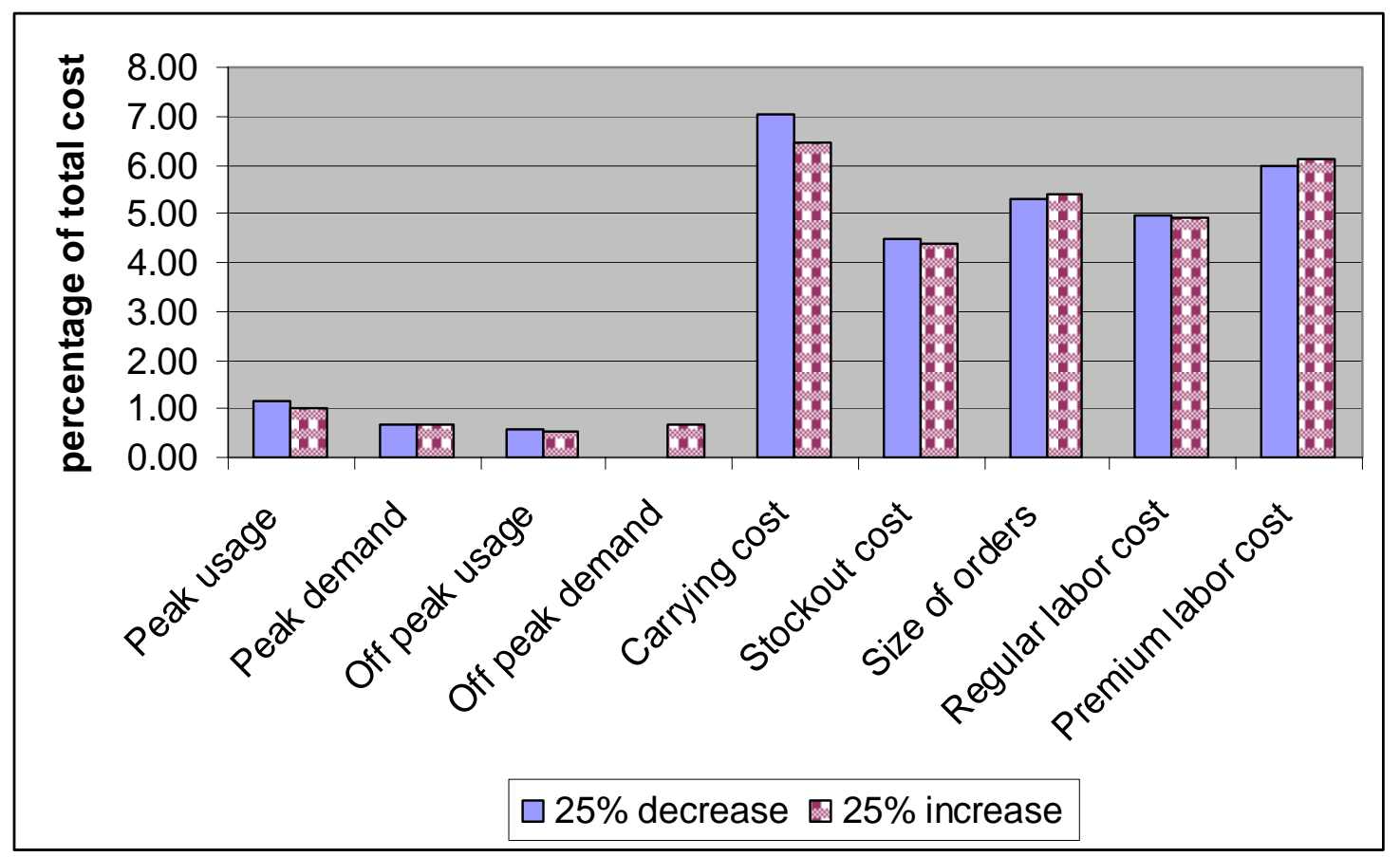

Figure 5.1 Sensitivity Analysis - max. of 2 lines operating

Figure 5.1 illustrates the percentage increase in the overall cost per year when each of parameter are increased or decreased by $25 \%$ while keeping the other parameters constant.

\subsubsection{Peak usage cost}

The total cost increased to 447,612, a $1 \%$ more than the original cost of 443,169 when the peak usage cost was increased by $25 \%$. When the peak usage cost was reduced by $25 \%$ to $\$ 0.06 / \mathrm{kWh}$, the cost per pallet was lowered by $1.13 \%$. Similarly in most cases, the difference in the percentage change when the parameters are increased or decreased by $25 \%$ is very small.

\subsubsection{Peak demand cost}

The total cost went up by $0.67 \%$ from the original cost to $\$ 446,119$, when the peak demand cost was increased by $25 \%$. Similarly, a $25 \%$ reduction in the peak demand cost led to a $0.7 \%$ reduction in the total cost. 


\subsubsection{Off-peak usage cost}

The total cost increased by $0.55 \%$ with a $25 \%$ increase in the off-peak usage cost. But the overall cost is reduced by $0.55 \%$ with a $25 \%$ reduction in the off-peak usage cost.

\subsubsection{Off-peak demand cost}

The total cost increased by $0.67 \%$ when the off-peak demand cost was set at $25 \%$ of the peak demand cost (\$3.50). Initially, there was no off-peak demand charge.

\subsubsection{Carrying cost}

A 25\% increase in the carrying costs of the WIP and finished goods inventory resulted in a $6.46 \%$ increase in the total cost. A reduction of was of $7 \%$ was observed when the costs were reduced by $25 \%$. The effect of carrying cost is more pronounced when 3 or 4 lines are operated due to the large number of products in the inventory.

\subsubsection{Stockout cost}

A $25 \%$ increase in stockout cost increases the overall cost by $4.38 \%$. A $25 \%$ decrease causes a $4.38 \%$ reduction in the cost. Since operating only two lines causes more stockouts, it affects the overall cost more. Though the stockout is varies a lot, this percentage gives the user a range of values to expect when the stockout cost is affected.

\subsubsection{Size of orders}

Originally, the size of the orders were in the form of a triangular distribution TRIA $(40,50,60)$. A $25 \%$ increase in this size increases the cost by $5.38 \%$. A $25 \%$ reduction leads to a $5.3 \%$ reduction in the overall cost.

\subsubsection{Regular labor cost}

If the regular (peak shift) labor cost is raised by $25 \%$, the overall cost is increased by $4.93 \%$. A $4.97 \%$ reduction was observed when the regular labor cost was cut by $25 \%$. 


\subsubsection{Premium labor cost}

A 25\% increase in the premium (off-peak) labor cost increased the overall cost by $6.13 \%$. There was a similar reduction of the overall cost by $5.99 \%$ with a $25 \%$ reduction of the premium labor cost.

Similar runs were performed with a maximum of three lines being operated simultaneously. The following results were obtained.

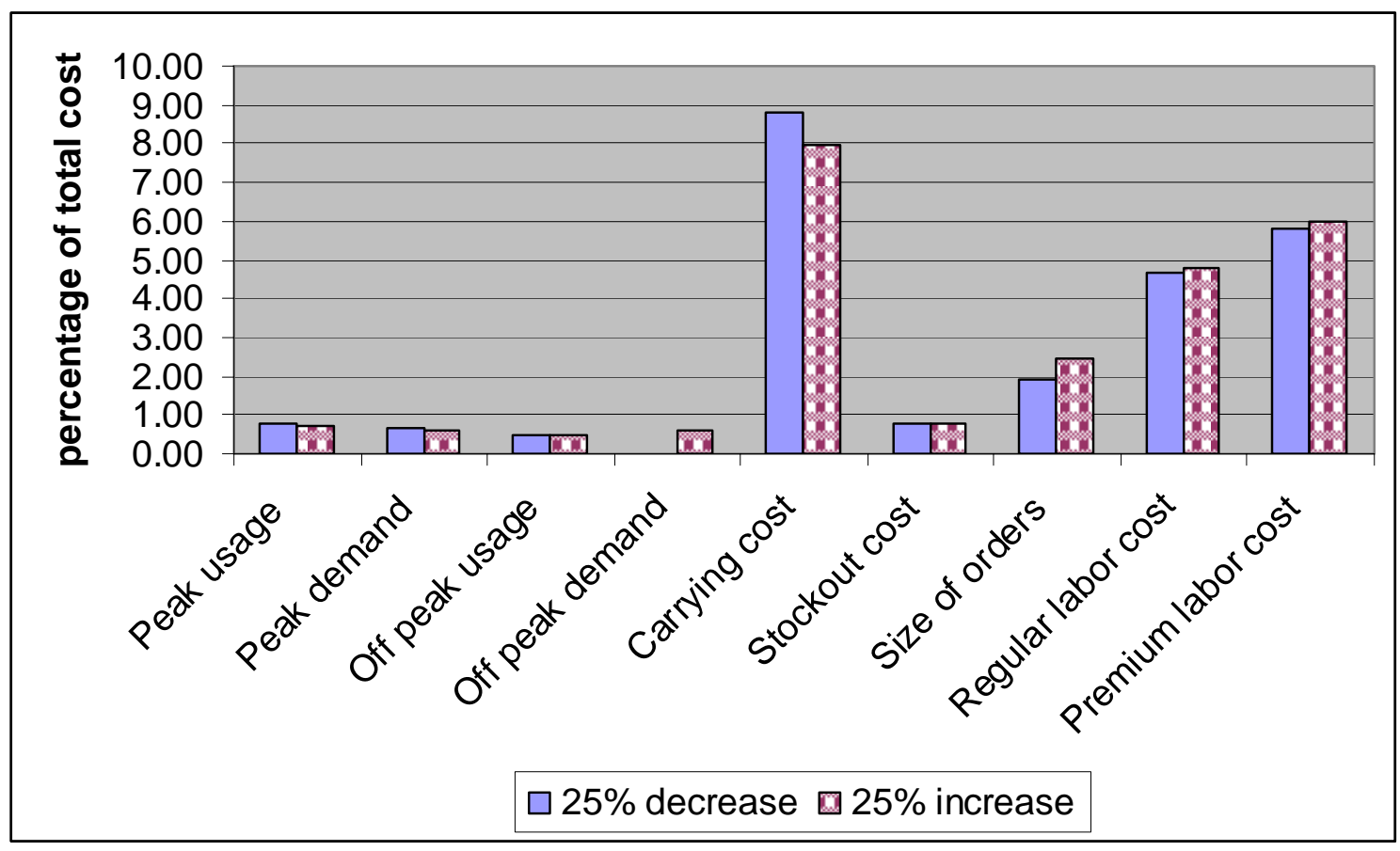

Figure 5.2 Sensitivity Analysis - max of 3 lines operating

Again, it can be seen that more impact is seen when the carrying cost or the labor cost is changed. But the level of impact varies with the number of lines being operated. There are more products and more worker-hours involved when more lines are operated. Therefore, the importance of inventory and labor also increases. Though the kWh becomes higher, its effect on the overall cost is marginal. The effect of size of the orders and the number of stockouts are less pronounced as the number of lines increases. But it is not very different between 3 or 4 lines because of the number of finished products produced is controlled by the limited capacity of the finishing line. 


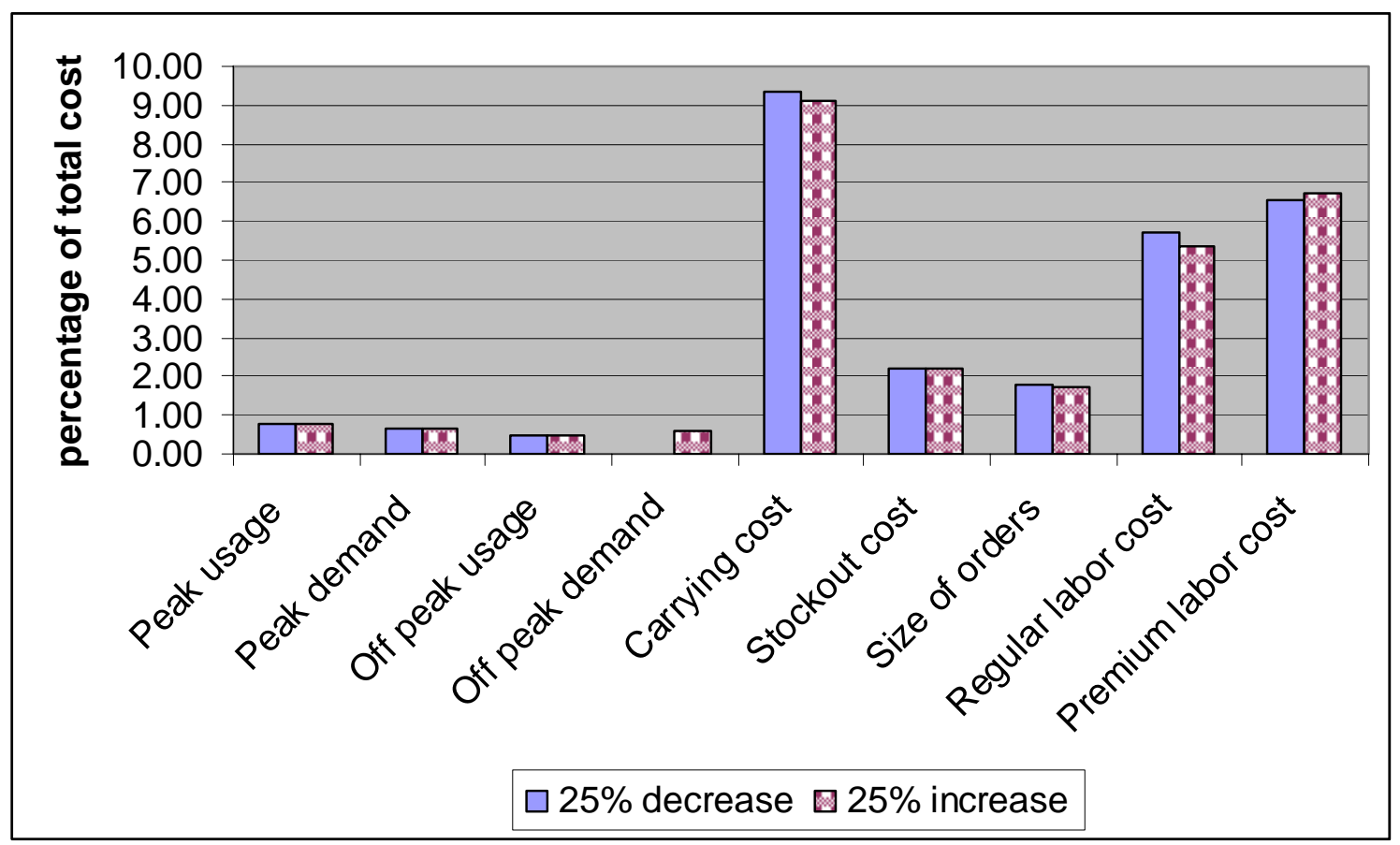

Figure 5.3 Sensitivity Analysis - max. of 4 lines operating

A similar trend to what was seen when the number of lines operated was changed from 2 to 3 is seen in figure 5.3. The effect of labor cost and carrying cost become more important than when three lines were operated. The effect of stockout and size of orders is lower than when only two lines were operated. These figures give the user an idea of the risk that he is taking when he implements a demand reduction strategy.

\subsection{Conclusion}

The analysis of the results obtained from running the model with modified inputs showed the effect of different parameters and the extent of those effects. It was found that for this particular scenario chosen, the carrying cost and the labor cost made a bigger impact than the other parameters. Numerous different combinations of various parameters can be verified depending on which of the factors the user considers important. The results explained in the previous sections also helped verify the model. Most of the results were close to what was expected based on the understanding of the model. 


\section{Chapter 6 \\ Conclusion and Future Work}

\subsection{Conclusion}

This research suggests that cutting down on electric demand does not always mean cutting down on costs. The effect of a demand reduction measures can vary widely from one operation to the other based on various different factors. This research was an attempt to emphasize this point and show that a software based approach will be helpful in identifying the factors involved and their impact.

Once such a software program customized to represent a specific operation is in place, the plant manager can get an idea of how his decisions will affect the cost of a product. This is especially true in operations where there is a lot of uncertainty and randomness. Even in more consistent operations, it provides a tool to see how the system performs after a measure has been implemented. It can be used as a tool to convince the management before implementing any measure. It makes more sense to see what happens using a computerized model of a system than actually trying it on the shop floor. This is probably one reason where we hear about cases where managers wanting to simulate their facilities but not really caring about the final results.

\subsection{Future Work}

Even the advanced demand controllers available in the market are limited in their function to disconnect non-essential equipment in a certain order if the electric demand goes beyond a preset limit. The real cost savings lies in involving process equipment in load staggering measures. But a tool is required to convince people about how the various costs will be affected if the process is modified.

Like any other work, there is scope for improvement in the future.

1. The software may be improved to incorporate energy uses of supporting systems such as the HVAC system, lighting, compressed air system etc. to get more accurate results. Since the operation and the energy use of these systems depend on the production and outdoor conditions, more factors come into play making the model quite complicated. It may be integrated with a knowledge 
base (an expert system) that can help suggesting ways to reduce cost based on the inputs given by the user.

2. Other forms of energy that may be used in a manufacturing setting may also be included to get a better idea of the costs involved. This may be a good idea for facilities that are considering fuel switch as an option.

3. A tool that can apply the simulation model of a manufacturing facility to find out which rate schedule will be the most suitable or suggest what kind of a schedule may be negotiated with the utility company may be an option. Sometimes the economics of an energy conservation measure may not be attractive if there is a change in the rate structure. In a deregulated environment where the user can decide the electricity supplier, the tool can be designed to search the most suitable one from a database of rate schedules.

4. Although model validation was attempted, a more extensive effort may be required to investigate the results. 


\section{References}

1. Allegheny Power, www.alleghenypower.com., 2002.

2. Abraham, S., Secretary of Energy, DOE News Release, December 13, 2001

3. Achariyaviriya, A., Tiansuwan, J., Soponronnarit, S., "Energy Optimization of Whole Longan Drying: Simulation Results”, International Journal of Ambient Energy, v 23, n 4, pp 212-220, Oct 2002.

4. American Council for an Energy Efficient Economy, Martin, K., Vine, E. and York, D., "Energy Efficiency and Electric System Reliability: A Look at ReliabilityFocused Energy Efficiency Programs Used to Help Address the Electricity Crisis of 2001”, May 2002.

5. Bureau of Economic Analysis, www.bea.doc.gov/, 2003.

6. Childress, R., "Closed Loop Energy Management Control of Large Industrial Facilities”, Energy Engineering, v 100, n 1, pp 17, 2003.

7. Chillarige, R., "Development of Expert Systems in Heating, Ventilating and Airconditioning (HVAC)- An energy approach”, West Virginia University,1999.

8. Creese, R., Adithan, M., and Pabla, B.S., "Estimating and Costing for the Metal Manufacturing Industries”, Marcel Dekker, 1992.

9. El-Sebaey, N., Yousef M.T, and El-Alayly A.A, "An Application of Expert System for Demand Side Management”, IEE Conference Publication, n 488, 2002.

10. Feldmann, K., Wunderlich, J., "Simulation Based Costing in Production Systems", Institute for Manufacturing Automation and Production Systems, http://www.costsim-consulting.de/sbc_wunderlich.pdf, 2001.

11. Fernandes, M., Capehart, B., and Capehart, L., "Allocation of Energy Costs in Manufacturing Using Activity Based Costing," Energy Engineering, v 94, n 4, pp 1733, Fall 1997.

12. "Ford Brazil: Designs Engine Plant from the Ground Up", http://www.arenasimulation.com/pdf/fiesta.pdf, 2003.

13. Gopalakrishnan B., Plummer R.W., Alkadi N.M., "Analysis of Energy Conservation Opportunities in Glass Manufacturing Facilities”, Energy Engineering, v 98, n 6, pp 27-48, 2001.

14. Heslin, K., "Conservation Always Reduced Cost", Energy User News. www.energyusernews.com/eun/cda/articleinformation/features/bnp features _item/ 0,2584,100894,00.html, 2002.

15. Joel, M., “Green and Purple Money”, Energy User News, www.energyusernews.com, 2003.

16. Kelton, D., Sadowski, R., and Sadowski, D., "Simulation with Arena”, McGraw Hill, $2^{\text {nd }}$ ed., 2002

17. Levine, D., and Sonnenblick, R., "On the Assessment of Utility Demand-Side Management Programs”, Energy Policy, v 22, n 10, p 848-856, Oct 1994. 
18. Mate, A., "Energy Analysis and Diagnostics in Wood Manufacturing Industry", Master's Thesis, West Virginia University, 2002.

19. Nilsson, H., "Many Faces of Demand-Side Management", Power Engineering Journal, v 8, n 5, , p 207-210, Oct 1994.

20. Norland D., Lind L., “Corporate Energy Management”, Energy Engineering, v 98, n 2, pp 53-66, 2000.

21. Selvaraj, R., "Plant-wide Energy Assessment using a Systems Approach”, Master's Thesis, West Virginia University, 2003.

22. Shannon, R.E., Long, S.S., Buckles, B.P., "Operation Research Methodologies in Industrial Engineering”, AIIE Transactions, vol. 12, pp. 364-367, 1980.

23. Smith, C, “Energy Management Principles”, Pergamon Press, New York, 1981.

24. Smith, W., “Industrial Process and Energy Efficiency”, Energy User News, Oct 2000.

25. Takakuwa, S., "The Use of Simulation in Activity Based Costing for Flexible Manufacturing Systems”, Proceedings of the 1997 Winter Simulation Conference.

26. Thomas, G., DaCosta J., "A Sample Survey of Corporate Operations Research”, Interfaces, v 9, pp 102-111, 1979.

27. Thumann, A., "Handbook of Energy Audits", The Fairmont Press, Georgia, $5^{\text {th }}$ Edition, 1998.

28. Turner, W., "Energy Management Handbook”, Fairmont Press, GA, 2001.

29. U.S. Department of Energy, http://www.energy.gov/, 2003.

30. Washington State University Cooperative Extension Energy Program, "Energy Efficiency Fact Sheet”, 2003 